\title{
Generalization of the Kermack-McKendrick SIR Model to a Patchy Environment for a Disease with Latency
}

\author{
J. Li* and X. Zou ${ }^{\dagger}$ \\ Department of Applied Mathematics University of Western Ontario \\ London, Ontario, Canada N6A 5B7
}

\begin{abstract}
In this paper, with the assumptions that an infectious disease has a fixed latent period in a population and the latent individuals of the population may disperse, we reformulate an SIR model for the population living in two patches (cities, towns, or countries etc.), which is a generalization of the classic Kermack-McKendrick SIR model. The model is given by a system of delay differential equations with a fixed delay accounting for the latency and non-local terms caused by the mobility of the individuals during the latent period. We analytically show that the model preserves some properties that the classic Kermack-McKendrick SIR model possesses: the disease always dies out, leaving a certain portion of the susceptible population untouched (called final sizes). Although we can not determine the two final sizes, we are able to show that the ratio of the final sizes in the two patches is totally determined by the ratio of the dispersion rates of the susceptible individuals between the two patches. We also explore numerically the patterns by which the disease dies out, and find that the new model may have very rich patterns for the disease to die out. In particular, it allows multiple outbreaks of the disease before it goes to extinction, strongly contrasting to the classic Kermack-McKendrick SIR model.
\end{abstract}

Key words: infectious disease, SIR model, latent period, patch, non-local infection, dispersion, multiple outbreaks

AMS subject classification: 34K18, 34K20, 34D 23, 37N25, 92D30

\footnotetext{
${ }^{*}$ Current address: Department of Mathematics and Statistics, University of Ottawa, Ottawa, ON K1N 6N5,Canada.

†Corresponding author. E-mail: xzou@uwo.ca
} 


\section{Introduction}

Kermak-McKendrick model [7] is one of the earliest triumphs in mathematical epidemiology [4]. This simple model is formulated for a population being divided into three disjoint sub-populations or compartments-susceptible class $S$, infective class $I$ and removed class $R$, and is given by the following system of ordinary differential equations

$$
\left\{\begin{array}{l}
\frac{d S}{d t}=-\lambda S I \\
\frac{d I}{d t}=\lambda S I-\sigma I
\end{array}\right.
$$

with the $R$ class being determined by $\frac{d R}{d t}=\sigma I$. Here the removed class could include the recovered individuals with permanent immunity, as well as the individuals who die of the disease in the case that the disease causes deaths. The constant $\sigma>0$ is called the removal rate and the constant $\lambda>0$ is called the infection rate. Given $S(0)=S_{0}>0$ and $I(0)=I_{0}>0$, the analysis of (1.1) has shown (see, e.g., $[5,11])$ that

(i) when $\mathcal{R}_{0}:=\lambda S_{0} / \sigma<1$, there is no outbreak of the disease in the sense that the population of the infectious class $I(t)$ decreases monotonically to 0 ;

(ii) when $\mathcal{R}_{0}:=\lambda S_{0} / \sigma>1$, there will be a single outbreak of the disease in the sense that $I(t)$ firstly increases monotonically to a maximum value, and after that, $I(t)$ decreases monotonically to 0 ;

(iii) in either of the above two situations, the disease eventually dies out of the population, leaving part of the population, denoted by $S_{\infty}$, untouched by the disease.

The quantity $\mathcal{R}_{0}$ defined above is referred to as the basic reproduction number of the SIR model (1.1), accounting for the average number of new infections that a single infectious individual can cause during the infection life time (see, e.g., $[1,5]$ ). The untouched part $S_{\infty}$ of the susceptible class is often referred to as the final size of the SIR model (1.1), and is determined implicitly by the equation

$$
I_{0}+S_{0}-\frac{\sigma}{\lambda} \ln S_{0}=S_{\infty}-\frac{\sigma}{\lambda} \ln S_{\infty} .
$$

Since $I_{0}$ is usually very small, this equation is approximated by

$$
S_{0}-\frac{\sigma}{\lambda} \ln S_{0}=S_{\infty}-\frac{\sigma}{\lambda} \ln S_{\infty}
$$

From the above summary for the model (1.1), we know that the disease dynamics of this model is very clear: the disease either dies out quickly without causing new infectious, or experiences a single outbreak before dying out. The final size $S_{\infty}$ and the magnitude of the outbreak (if any) depend on the initial susceptible population size $S_{0}$. Obviously, this model does not include demographic structure and is suitable for describing those diseases that suddenly develop in a community and then disappear without infecting the entire community. 
In this paper, we aim to modify the model (1.1) to include two important factors: latency and spatial mobility. The scenario for the former is the fact that many diseases have latent periods, namely, an individual infected by a disease will not be infectious until some time after the infection. Among such diseases are Hepatitis B and tuberculosis including bovine tuberculosis, which may take months to develop to infectious stage, as well as some childhood diseases such as measles, chicken pox, and whooping cough (pertussis), etc.(see Table 3.1 in [1]). For a disease with latency, it is reasonable to introduce into the model one more class: the exposed or latent class denoted by $L(t)$, consisting of those individuals that have been infected but are not yet infectious. In such a situation, the infection term $\lambda S(t) I(t)$ would not contribute directly to $I^{\prime}(t)$; instead it should contribute to $L^{\prime}(t)$. The inclusion of mobility in the model can be easily justified by the fact that the world has become highly connected nowadays, and travels between patches (towns, cities and countries) have become more important and common, and sometimes even inevitable.

Along the above lines, there arise two basic questions: (Q1) how to incorporate these two factors into model (1.1)? (Q2) what are the consequences? As an initial attempt, in this paper we only consider a simple situation for these two factors: a fixed latent period and two patches. Under these two assumptions, we derive, in Section 2, a new model which is a modification of (1.1). The new model carries a discrete delay and non-local infection terms caused by the mobility of latent individuals. Roughly speaking, such non-local terms explain the fact that an individual infected in one of the two patches may be, due to his/her mobility, in either patch with certain probability at the time when this individual becomes infectious. In Section 3, we are able to address the well-posedness of the model by proving the positivity and boundedness of solutions. By employing the theory of asymptotically autonomous systems, we are also able to show that, like in (1.1), the infectious populations in both patches will go to extinction, implying that the disease will eventually die out in both patches. Although we can not obtain two equations that determine the two final sizes $S_{1}(\infty)$ and $S_{2}(\infty)$, we are able to prove that the ratio of these two final sizes are fully determined by the ratio of the dispersion rates of susceptible individuals between the two patches. The presence of the latent delay and the non-local infection terms in the model makes it very difficult to obtain a complete description of the patterns by which the disease dies out. However, our numeric study in Section 4 shows that the new model may demonstrate very rich patterns by which the disease dies out. Particularly, multiple outbreaks may occur in the new model. This important finding is in contrast to the disease outbreak patterns described by the classic Kermack-McKendrick model (1.1), and may provide some insights into the spread of diseases that do develop multiple outbreaks in reality. In Section 5, we summarize our results and discuss their biological implications; we also compare our model with some existing ones and discuss possible future extension of the model to include a demographic structure.

We emphasize that the goal of this paper is to extend the classical Kermack-McKendrick model (1.1), which does not allow the persistence of the disease, to a model for a disease with a fixed latent period spreading in a patchy environment. Thus, our model also does not support a disease becoming endemic. We point out that there have been numerous models that describe the disease dynamics over patchy environments and that can support diseases either going to extinction or becoming endemic, among which are $[2,3,8,12,14,15,16]$ and the references therein. However, 
these models do not consider a fixed latent period, neither do they contain non-local infection terms which are exactly the novelty of our model (2.15).

\section{Derivation of the model}

Consider a population that lives in two patches. Let $S_{i}(t), I_{i}(t), R_{i}(t)$ be, respectively, the subpopulations of the susceptible, infectious and removed classes in patch $i, i=1,2$ at time $t$. The two patches are connected in the sense that individuals can move between these two patches. Assume that the disease has a fixed latent period, denoted by $\tau$. Due to this latency and the mobility of the individuals during the latent period, the rate at which patch 1 (patch 2) gains new infectious at time $t$ depends on the new infections infected $\tau$ time units ago not only in patch 1 (patch 2) but also in patch 2 (patch 1). To determine such a dependence, we use the notion of infection age, denoted by $a$. Let $l_{i}(t, a)$ be the density (with respect to the infection age $a$ ) of individuals at time $t$ in patch $i(i=1,2)$ with infection age $a$. Similar to the population with natural age structure (see Diekmann and Metz [9]), $l_{1}(t, a)$ and $l_{2}(t, a)$ are governed by the following first-order partial differential equations:

$$
\left\{\begin{array}{l}
\frac{\partial l_{1}(t, a)}{\partial t}+\frac{\partial l_{1}(t, a)}{\partial a}=-d_{1}(a) l_{1}(t, a)+D_{2}(a) l_{2}(t, a)-D_{1}(a) l_{1}(t, a) \\
\frac{\partial l_{2}(t, a)}{\partial t}+\frac{\partial l_{2}(t, a)}{\partial a}=-d_{2}(a) l_{2}(t, a)+D_{1}(a) l_{1}(t, a)-D_{2}(a) l_{2}(t, a) .
\end{array}\right.
$$

Here $D_{j}(a) l_{j}(t, a)$ corresponds to the dispersal of the individuals at the infection age $a$ from patch $j$ to patch $i$, where, $1 \leq i \neq j \leq 2$, and $d_{i}(a)$ represents the rate at which the infected individuals in patch $i$ is removed via deaths due to the disease, recovery after treatments with permanent immunity, and other possible means such as isolation or quarantine. In addition, we have assumed that there is no delay in the dispersal between patches and there is no loss during migration from patch $j$ to patch $i$, meaning that all of those who leave patch $j$ arrive at patch $i$ safely. Obviously, if the two patches are too far away from each other, this assumption should be re-examined.

By the meaning of $l_{i}(t, a)$, it is obvious that at a given time $t$, the number of the infectious individuals in patch $i$ is given by

$$
I_{i}(t)=\int_{\tau}^{\infty} l_{i}(t, a) d a .
$$

Obviously $l_{i}(t, 0)$ corresponds to new infections which come from direct contacts between infectious and susceptible individuals. Mass action infection mechanism leads to

$$
l_{i}(t, 0)=\lambda_{i} I_{i}(t) S_{i}(t) .
$$

where $\lambda_{i}$ is the the infection rate in patch $i, i=1,2$. It is also biologically reasonable to assume

$$
l_{i}(t, \infty)=0 .
$$


We point out that (2.4) holds if we assume that $d_{i}(a), i=1,2$, are bounded away from zero for large $a$ (as is assumed in (2.5)). Indeed, using the method of characteristics, one can solve (2.1) and (2.3) with a given initial condition and directly verify that the solution satisfies (2.4).

For convenience of showing the main idea to build up the new model, we further assume that

$$
d_{i}(a)=\left\{\begin{array}{ccc}
d_{l}(a), & \text { for } 0 \leq a \leq \tau, & \text { and } i=1,2, \\
d_{I_{i}}(a)=\gamma_{i}+\mu_{i}, & \text { for } \quad a>\tau, & \text { and } i=1,2,
\end{array}\right.
$$

and

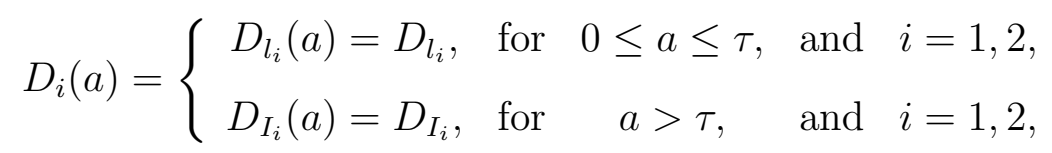

where $d_{l}(a)$ denotes the removal rate of latent individuals due to possible means such as quarantine or isolation, and it is assumed to be independent on the patch, and $\gamma_{i}$ represents the removal rate of infectious individuals accounting for recovery with permanent immunity and possible isolation or quarantine in patch $i, i=1,2$, and $\mu_{i}$ corresponds to the disease mortality rate of infectious individuals in patch $i, i=1,2$. For simplicity of notation, in the sequel, we let $\sigma_{i}=\gamma_{i}+\mu_{i}$, $i=1,2$.

By integrating (2.1) with respect to $a$ from $\tau$ to $\infty$, we have

$$
\begin{aligned}
\frac{d I_{i}(t)}{d t}= & -\int_{\tau}^{\infty} \frac{\partial l_{i}(t, a)}{\partial a} d a-\int_{\tau}^{\infty} d_{I_{i}}(a) l_{i}(t, a) d a \\
& +\int_{\tau}^{\infty} D_{I_{j}}(a) l_{j}(t, a) d a-\int_{\tau}^{\infty} D_{I_{i}}(a) l_{i}(t, a) d a \\
= & l_{i}(t, \tau)-\sigma_{i} I_{i}(t)+D_{I_{j}} I_{j}(t)-D_{I_{i}} I_{i}(t),
\end{aligned}
$$

for $i, j=1,2$ with $j \neq i$. The equations governing $S_{i}(t)$ and $R_{i}(t)$ are given in the usual way, which, together with (2.7), lead to the following model under the above assumptions:

$$
\left\{\begin{array}{l}
\frac{d S_{1}(t)}{d t}=D_{S_{2}} S_{2}(t)-D_{S_{1}} S_{1}(t)-\lambda_{1} I_{1}(t) S_{1}(t) \\
\frac{d S_{2}(t)}{d t}=D_{S_{1}} S_{1}(t)-D_{S_{2}} S_{2}(t)-\lambda_{2} I_{2}(t) S_{2}(t) \\
\frac{d I_{1}(t)}{d t}=-\sigma_{1} I_{1}(t)+D_{I_{2}} I_{2}(t)-D_{I_{1}} I_{1}(t)+l_{1}(t, \tau) \\
\frac{d I_{2}(t)}{d t}=-\sigma_{2} I_{2}(t)+D_{I_{1}} I_{1}(t)-D_{I_{2}} I_{2}(t)+l_{2}(t, \tau) \\
\frac{d R_{1}(t)}{d t}=\int_{0}^{\tau} d_{l}(a) l_{1}(t, a) d a+\sigma_{1} I_{1}(t)+D_{R_{2}} R_{2}(t)-D_{R_{1}} R_{1}(t) \\
\frac{d R_{2}(t)}{d t}=\int_{0}^{\tau} d_{l}(a) l_{2}(t, a) d a+\sigma_{2} I_{2}(t)+D_{R_{1}} R_{1}(t)-D_{R_{2}} R_{2}(t) .
\end{array}\right.
$$

where $D_{S_{i}} \geq 0$ is the rate at which susceptible individuals migrate from patch $i$ to patch $j(j \neq i)$, and $D_{R_{i}} \geq 0$ is the rate at which removed individuals migrate from patch $i$ to patch $j(j \neq i)$. 
Note that as in the classic Kermack-McKendrick model (1.1), the two equations for the removed classes $R_{1}$ and $R_{2}$ are decoupled from the equations for $S_{1}, S_{2}, I_{1}$ and $I_{2}$, thus we only need to consider the first four equations in (2.8). The remaining work in this section is to evaluate the term $l_{i}(t, \tau)$ for $i=1,2$ in terms of $S_{i}$ and $I_{i}(i=1,2)$. To this end, we fix $s \geq 0$ and let

$$
V_{i}^{s}(t)=l_{i}(t, t-s), \text { for } s \leq t \leq s+\tau \text { and } i=1,2 .
$$

Then, for $1 \leq i \neq j \leq 2$,

$$
\begin{aligned}
\frac{d}{d t} V_{i}^{s}(t) & =\left.\frac{\partial}{\partial t} l_{i}(t, a)\right|_{a=t-s}+\left.\frac{\partial}{\partial a} l_{i}(t, a)\right|_{a=t-s} \\
& =-d_{i}(t-s) l_{i}(t, t-s)+D_{j}(t-s) l_{j}(t, t-s)-D_{i}(t-s) l_{i}(t, t-s) \\
& =-d_{l}(t-s) V_{i}^{s}(t)+D_{l_{j}}(t-s) V_{j}^{s}(t)-D_{l_{i}}(t-s) V_{i}^{s}(t)
\end{aligned}
$$

Since $s \leq t \leq s+\tau$, we have

$$
\frac{d}{d t}\left(V_{1}^{s}(t)+V_{2}^{s}(t)\right)=-d_{l}(t-s)\left(V_{1}^{s}(t)+V_{2}^{s}(t)\right) .
$$

Solving this linear equation and using the condition (2.3), we get

$$
\begin{aligned}
V_{1}^{s}(t)+V_{2}^{s}(t) & =e^{-\int_{s}^{t} d_{l}(\theta-s) d \theta}\left(V_{1}^{s}(s)+V_{2}^{s}(s)\right) \\
& =e^{-\int_{s}^{t} d_{l}(\theta-s) d \theta}\left(l_{1}(s, 0)+l_{2}(s, 0)\right) \\
& =e^{-\int_{s}^{t} d_{l}(\theta-s) d \theta}\left(\lambda_{1} I_{1}(s) S_{1}(s)+\lambda_{2} I_{2}(s) S_{2}(s)\right) \\
& =e^{-\int_{0}^{t-s} d_{l}(a) d a}\left(\lambda_{1} I_{1}(s) S_{1}(s)+\lambda_{2} I_{2}(s) S_{2}(s)\right), \text { for } s \leq t \leq s+\tau
\end{aligned}
$$

Hence, from (2.9),

$$
\begin{aligned}
\frac{d}{d t} V_{1}^{s}(t)= & -d_{l}(t-s) V_{1}^{s}(t)+D_{l_{2}}(t-s) V_{2}^{s}(t)-D_{l_{1}}(t-s) V_{1}^{s}(t) \\
= & -d_{l}(t-s) V_{1}^{s}(t)+D_{l_{2}}(t-s)\left(V_{1}^{s}(t)+V_{2}^{s}(t)\right)-\left(D_{l_{1}}(t-s)+D_{l_{2}}(t-s)\right) V_{1}^{s}(t) \\
= & -\left(d_{l}(t-s)+D_{l_{1}}(t-s)+D_{l_{2}}(t-s)\right) V_{1}^{s}(t) \\
\quad & \quad+D_{l_{2}}(t-s) e^{-\int_{0}^{t-s} d_{l}(a) d a}\left(\lambda_{1} I_{1}(s) S_{1}(s)+\lambda_{2} I_{2}(s) S_{2}(s)\right) \\
& \quad-D^{*}(t-s) V_{1}^{s}(t)+D_{l_{2}}(t-s) e^{-\int_{0}^{t-s} d_{l}(a) d a}\left(\lambda_{1} I_{1}(s) S_{1}(s)+\lambda_{2} I_{2}(s) S_{2}(s)\right)
\end{aligned}
$$

where $D^{*}(a) \triangleq d_{l}(a)+D_{l_{1}}(a)+D_{l_{2}}(a)$. This is a first-order linear inhomogeneous equation for 
$V_{1}^{s}(t)$, the solution of which is given, using (2.3), by

$$
\begin{aligned}
V_{1}^{s}(t)= & e^{-\int_{s}^{t} D^{*}(\theta-s) d \theta} V_{1}^{s}(s) \\
& +\int_{s}^{t} e^{-\int_{\xi}^{t} D^{*}(\theta-s) d \theta} D_{l_{2}}(\xi-s) e^{-\int_{0}^{\xi-s} d_{l}(a) d a} d \xi\left(\lambda_{1} I_{1}(s) S_{1}(s)+\lambda_{2} I_{2}(s) S_{2}(s)\right) \\
= & e^{-\int_{0}^{t-s} D^{*}(a) d a} \lambda_{1} I_{1}(s) S_{1}(s) \\
& +\int_{s}^{t} e^{-\int_{\xi-s}^{t-s} D^{*}(a) d a} D_{l_{2}}(\xi-s) e^{-\int_{0}^{\xi-s} d_{l}(a) d a} d \xi\left(\lambda_{1} I_{1}(s) S_{1}(s)+\lambda_{2} I_{2}(s) S_{2}(s)\right) .
\end{aligned}
$$

Let $\hat{D}(a)=D_{l_{1}}(a)+D_{l_{2}}(a)$. Then

$$
\begin{aligned}
& l_{1}(t, \tau)= V_{1}^{t-\tau}(t) \\
&=\left(e^{-\int_{0}^{\tau} D^{*}(a) d a}+\int_{t-\tau}^{t} e^{-\int_{\xi-t+\tau}^{\tau} D^{*}(a) d a} D_{l_{2}}(\xi-t+\tau) e^{-\int_{0}^{\xi-t+\tau} d_{l}(a) d a} d \xi\right) \lambda_{1} I_{1}(t-\tau) S_{1}(t-\tau) \\
& \quad+\left(\int_{t-\tau}^{t} e^{-\int_{\xi-t+\tau}^{\tau} D^{*}(a) d a} D_{l_{2}}(\xi-t+\tau) e^{-\int_{0}^{\xi-t+\tau} d_{l}(a) d a} d \xi\right) \lambda_{2} I_{2}(t-\tau) S_{2}(t-\tau) \\
&=\left(e^{-\int_{0}^{\tau} D^{*}(a) d a}+e^{-\int_{0}^{\tau} d_{l}(a) d a} \int_{0}^{\tau} e^{-\int_{\theta}^{\tau} \hat{D}(a) d a} D_{l_{2}}(\theta) d \theta\right) \lambda_{1} I_{1}(t-\tau) S_{1}(t-\tau) \\
& \quad+\left(e^{-\int_{0}^{\tau} d_{l}(a) d a} \int_{0}^{\tau} e^{-\int_{\theta}^{\tau} \hat{D}(a) d a} D_{l_{2}}(\theta) d \theta\right) \lambda_{2} I_{2}(t-\tau) S_{2}(t-\tau) \\
&= e^{-\int_{0}^{\tau} d_{l}(a) d a}\left(1-\int_{0}^{\tau} e^{-\int_{\theta}^{\tau} \hat{D}(a) d a} D_{l_{1}}(\theta) d \theta\right) \lambda_{1} I_{1}(t-\tau) S_{1}(t-\tau) \\
& \quad+\left(e^{-\int_{0}^{\tau} d_{l}(a) d a} \int_{0}^{\tau} e^{-\int_{\theta}^{\tau} \hat{D}(a) d a} D_{l_{2}}(\theta) d \theta\right) \lambda_{2} I_{2}(t-\tau) S_{2}(t-\tau) .
\end{aligned}
$$

Thus,

$$
l_{1}(t, \tau)=\epsilon\left(1-\alpha_{1}(\tau)\right) \lambda_{1} I_{1}(t-\tau) S_{1}(t-\tau)+\epsilon \alpha_{2}(\tau) \lambda_{2} I_{2}(t-\tau) S_{2}(t-\tau),
$$

where

$$
\epsilon=e^{-\int_{0}^{\tau} d_{l}(a) d a}, \quad \alpha_{1}(\tau)=\int_{0}^{\tau} e^{-\int_{\theta}^{\tau} \hat{D}(a) d a} D_{l_{1}}(\theta) d \theta, \quad \alpha_{2}(\tau)=\int_{0}^{\tau} e^{-\int_{\theta}^{\tau} \hat{D}(a) d a} D_{l_{2}}(\theta) d \theta .
$$

Similarly,

$$
l_{2}(t, \tau)=\epsilon\left(1-\alpha_{2}(\tau)\right) \lambda_{2} I_{2}(t-\tau) S_{2}(t-\tau)+\epsilon \alpha_{1}(\tau) \lambda_{1} I_{1}(t-\tau) S_{1}(t-\tau) .
$$


Substituting (2.12) and (2.14) into (2.8) and taking out the first four equations for $S_{1}, S_{2}, I_{1}$ and $I_{2}$ results in the following new model:

$$
\left\{\begin{aligned}
& \frac{d S_{1}(t)}{d t}=D_{S_{2}} S_{2}(t)-D_{S_{1}} S_{1}(t)-\lambda_{1} I_{1}(t) S_{1}(t) \\
& \frac{d S_{2}(t)}{d t}=D_{S_{1}} S_{1}(t)-D_{S_{2}} S_{2}(t)-\lambda_{2} I_{2}(t) S_{2}(t) \\
& \frac{d I_{1}(t)}{d t}=-\sigma_{1} I_{1}(t)+D_{I_{2}} I_{2}(t)-D_{I_{1}} I_{1}(t) \\
& \quad+\epsilon\left(1-\alpha_{1}(\tau)\right) \lambda_{1} I_{1}(t-\tau) S_{1}(t-\tau)+\epsilon \alpha_{2}(\tau) \lambda_{2} I_{2}(t-\tau) S_{2}(t-\tau) \\
& \frac{d I_{2}(t)}{d t}=-\sigma_{2} I_{2}(t)+D_{I_{1}} I_{1}(t)-D_{I_{2}} I_{2}(t) \\
& \quad+\epsilon\left(1-\alpha_{2}(\tau)\right) \lambda_{2} I_{2}(t-\tau) S_{2}(t-\tau)+\epsilon \alpha_{1}(\tau) \lambda_{1} I_{1}(t-\tau) S_{1}(t-\tau)
\end{aligned}\right.
$$

From the above model, we see that the dispersion of latent individuals plays a different role from that of susceptible and infectious individuals. The explanation for those instantaneous terms are quite straightforward, and we now explain those delayed terms in the model. The probability that an individual infected in patch 1 can survive to the infection age $\tau$ (at which the infected individual becomes infectious) is $\epsilon$. Due to the mobility during the latent period between the two patches, $\tau$ time units later, a survived infected individual infected in patch 1 , may be in patch 1 with probability $\left(1-\alpha_{1}(\tau)\right)$ or in patch 2 with probability $\alpha_{1}(\tau)$. This explains the term $\epsilon(1-$ $\left.\alpha_{1}(\tau)\right) \lambda_{1} I_{1}(t-\tau) S_{1}(t-\tau)$ in $I_{1}$ equation and the term $\epsilon \alpha_{1}(\tau) \lambda_{1} I_{1}(t-\tau) S_{1}(t-\tau)$ in $I_{2}$ equation. The terms $\epsilon\left(1-\alpha_{2}(\tau)\right) \lambda_{2} I_{2}(t-\tau) S_{2}(t-\tau)$ in $I_{2}$ equation and the term $\epsilon \alpha_{2}(\tau) \lambda_{2} I_{2}(t-\tau) S_{2}(t-\tau)$ in $I_{1}$ equation are explained similarly. Alternatively, we may explain these terms in light of fractions as below. Among the individuals infected in the first patch $\tau$ time units ago, a fraction $\epsilon$ can survive to the infection age $\tau$, a fraction $\left(1-\alpha_{1}(\tau)\right)$ of which is now in patch 1 while a fraction $\alpha_{1}(\tau)$ is in patch 2 .

\section{Mathematical analysis}

Model (2.15) is a system of delay differential equations with non-local interactions over a twopatch environment. Firstly and naturally, as far as mathematical analysis of the system is concerned, initial conditions of delayed type should be considered. In other words, we consider the system with the following initial conditions:

$$
S_{i}(\theta)=\phi_{i}(\theta) \geq 0 \text {, and } I_{i}(\theta)=\psi_{i}(\theta) \geq 0, \text { for } i=1,2 \text {, and } \theta \in[-\tau, 0],
$$

where nonnegativity is based on biological consideration. By the fundamental theory of delay differential equations (see, e.g., Hale and Verduyn Lunel [6]), we know that for any given continuous functions $\phi_{i}(\theta), \psi_{i}(\theta), i=1,2$, the initial value problem (2.15) with (3.1) has a unique solution. In order for (2.15) with (3.1) to be biologically well-posed, we need to make sure that the solution remains nonnegative for $t \geq 0$ and is bounded. 
Theorem 1. Assume that $\phi_{i}(\theta)$ and $\psi_{i}(\theta), i=1,2$, are continuous. Then the solution of the initial value problem (2.15) with (3.1) remains nonnegative for $t \geq 0$ and is bounded.

Proof. Firstly, we show the nonnegativity of the solution. For this purpose, let us rewrite the system (2.15) as follows:

$$
\begin{aligned}
\frac{d}{d t} \mathbf{S}(t) & =M(t) \mathbf{S}(t) \\
\frac{d}{d t} \mathbf{I}(t) & =A \mathbf{I}(t)+B(t) \mathbf{I}(t-\tau),
\end{aligned}
$$

where $\mathbf{S}(t)=\left(S_{1}(t), S_{2}(t)\right)^{T}$ and $\mathbf{I}(t)=\left(I_{1}(t), I_{2}(t)\right)^{T}$ with $T$ representing the transpose of a vector, and

$$
\begin{gathered}
M(t) \triangleq\left[\begin{array}{cc}
-D_{S_{1}}-\lambda_{1} I_{1}(t) & D_{S_{2}} \\
D_{S_{1}} & -D_{S_{2}}-\lambda_{2} I_{2}(t)
\end{array}\right], \\
A \triangleq\left[\begin{array}{cc}
-\left(\sigma_{1}+D_{I_{1}}\right) & D_{I_{2}} \\
D_{I_{1}} & -\left(\sigma_{2}+D_{I_{2}}\right)
\end{array}\right], \\
B(t) \triangleq\left[\begin{array}{cc}
\epsilon\left(1-\alpha_{1}(\tau)\right) \lambda_{1} S_{1}(t-\tau) & \epsilon \alpha_{2}(\tau) \lambda_{2} S_{2}(t-\tau) \\
\epsilon \alpha_{1}(\tau) \lambda_{1} S_{1}(t-\tau) & \epsilon\left(1-\alpha_{2}(\tau)\right) \lambda_{2} S_{2}(t-\tau)
\end{array}\right] .
\end{gathered}
$$

Noting that the off-diagonal elements of matrix $M(t)$ are nonnegative, we conclude that the entries of the matrix $e^{\int_{0}^{t} M(\xi) d \xi}$ are all nonnegative. Indeed, let $h(t)=\max \left\{D_{S_{1}}+\lambda_{1} I_{1}(t)+1, D_{S_{2}}+\right.$ $\left.\lambda_{2} I_{2}(t)+1\right\}$ and rewrite $M(t)$ as

$$
M(t)=\left[\begin{array}{cc}
-h(t) & 0 \\
0 & -h(t)
\end{array}\right]+\left[\begin{array}{cc}
h(t)-D_{S_{1}}-\lambda_{1} I_{1}(t) & D_{S_{2}} \\
D_{S_{1}} & h(t)-D_{S_{2}}-\lambda_{2} I_{2}(t)
\end{array}\right] \triangleq-h(t) E+M_{1}(t),
$$

where $E$ is the $2 \times 2$ identity matrix. Then all entries of $M_{1}(t)$ are nonnegative, and hence, so are the entries of $e^{\int_{0}^{t} M_{1}(\xi) d \xi}$. Also

$$
e^{\int_{0}^{t}-h(\xi) E d \xi}=\left[\begin{array}{cc}
e^{-\int_{0}^{t} h(\xi) d \xi} & 0 \\
0 & e^{-\int_{0}^{t} h(\xi) d \xi}
\end{array}\right]
$$

Noting that the scalar matrix $-h(t) E$ commutes with any $2 \times 2$ matrix (hence with $M_{1}(t)$ ), we have

$$
e^{\int_{0}^{t} M(\xi) d \xi}=e^{\int_{0}^{t}-h(\xi) E d \xi} e^{\int_{0}^{t} M_{1}(\xi) d \xi},
$$

implying that all entries of $e^{\int_{0}^{t} M(\xi) d \xi}$ are nonnegative. Now, from the equation (3.2), we obtain

$$
\mathbf{S}(t)=e^{\int_{0}^{t} M(\xi) d \xi} \mathbf{S}(0) \geq 0, \text { for } t \geq 0,
$$


Similarly, for any $t \geq 0$, all entries of $e^{A t}$ are nonnegative. Moreover, by the nonnegativity of $S_{1}(t)$ and $S_{2}(t)$ established above, we know that all entries of $B(t)$ are all nonnegative. Now, (3.3) leads to

$$
\mathbf{I}(t)=e^{A t} \mathbf{I}(0)+\int_{0}^{t} e^{A(t-\xi)} B(\xi) \mathbf{I}(\xi-\tau) d \xi,
$$

implying $\mathbf{I}(t) \geq 0$ for $t \in[0, \tau]$ from the initial condition $I_{i}(\theta) \geq 0$ for $\theta \in[-\tau, 0]$ and $i=1,2$. This and (3.5) ensure $\mathbf{I}(t) \geq 0$ for $t \in[\tau, 2 \tau]$. By induction, we then conclude that $\mathbf{I}(t) \geq 0$ for $t \geq 0$.

Now, we show that $S_{i}(t)$ and $I_{i}(t)$ for $i=1,2$ are bounded. Let $N(t)=S_{1}(t)+I_{1}(t)+S_{2}(t)+$ $I_{2}(t)$. Direct calculation shows that

$$
\begin{aligned}
\frac{d}{d t} N(t)= & -\sigma_{1} I_{1}(t)-\sigma_{2} I_{2}(t)-\lambda_{1} I_{1}(t) S_{1}(t)-\lambda_{2} I_{2}(t) S_{2}(t) \\
& +\epsilon \lambda_{1} I_{1}(t-\tau) S_{1}(t-\tau)+\epsilon \lambda_{2} I_{2}(t-\tau) S_{2}(t-\tau) \\
\leq & -\sigma_{1} I_{1}(t)-\sigma_{2} I_{2}(t)-\lambda_{1} I_{1}(t) S_{1}(t)-\lambda_{2} I_{2}(t) S_{2}(t) \\
& +\lambda_{1} I_{1}(t-\tau) S_{1}(t-\tau)+\lambda_{2} I_{2}(t-\tau) S_{2}(t-\tau) \\
= & -\sigma_{1} I_{1}(t)-\sigma_{2} I_{2}(t)-\frac{d}{d t} \int_{t-\tau}^{t} \lambda_{1} I_{1}(\xi) S_{1}(\xi) d \xi-\frac{d}{d t} \int_{t-\tau}^{t} \lambda_{2} I_{2}(\xi) S_{2}(\xi) d \xi \\
= & -\sigma_{1} I_{1}(t)-\sigma_{2} I_{2}(t)-\frac{d}{d t} \int_{t-\tau}^{t}\left(\lambda_{1} I_{1}(\xi) S_{1}(\xi)+\lambda_{2} I_{2}(\xi) S_{2}(\xi)\right) d \xi .
\end{aligned}
$$

Hence,

$$
\frac{d}{d t}\left(N(t)+\int_{t-\tau}^{t}\left(\lambda_{1} I_{1}(\xi) S_{1}(\xi)+\lambda_{2} I_{2}(\xi) S_{2}(\xi)\right) d \xi\right) \leq-\sigma_{1} I(t)-\sigma_{2} I_{2}(t) \leq 0,
$$

implying

$$
N(t)+\int_{t-\tau}^{t}\left(\lambda_{1} I_{1}(\xi) S_{1}(\xi)+\lambda_{2} I_{2}(\xi) S_{2}(\xi)\right) d \xi \leq N(0)+\int_{-\tau}^{0}\left(\lambda_{1} I_{1}(\xi) S_{1}(\xi)+\lambda_{2} I_{2}(\xi) S_{2}(\xi)\right) d \xi .
$$

By nonnegativity of $S_{i}(t)$ and $I_{i}(t)(i=1,2)$, we have

$$
\begin{aligned}
N(t) & \leq N(0)+\int_{-\tau}^{0}\left(\lambda_{1} I_{1}(\xi) S_{1}(\xi)+\lambda_{2} I_{2}(\xi) S_{2}(\xi)\right) d \xi \\
& =S_{1}(0)+S_{2}(0)+I_{1}(0)+I_{2}(0)+\int_{-\tau}^{0}\left(\lambda_{1} I_{1}(\xi) S_{1}(\xi)+\lambda_{2} I_{2}(\xi) S_{2}(\xi)\right) d \xi \triangleq K
\end{aligned}
$$

Therefore,

$$
S_{i}(t), I_{i}(t) \leq K, i=1,2,
$$


where $K$ is the constant on the right-hand side of (3.6), depending on the initial values of susceptible and infectious individuals. The proof of Theorem 1 is completed.

Remark 2. From (3.4) and (3.5), we know that (i) if either $S_{1}(0)>0$ or $S_{2}(0)>0$, then both $S_{1}(t)$ and $S_{2}(t)$ remain strictly positive for all $t>0$; (ii) if either $I_{1}(0)>0$ or $I_{2}(0)>0$, then both $I_{1}(t)$ and $I_{2}(t)$ remain strictly positive for all $t>0$.

Next, we show that, as in the classic Kermack-McKendrick model, the $I_{1}(t)$ and $I_{2}(t)$ components of the solution to (2.15) with (3.1) always converge to zero.

Let $G(t)=S_{1}(t)+S_{2}(t)$. Then

$$
\frac{d}{d t} G(t)=-\left(\lambda_{1} I_{1}(t) S_{1}(t)+\lambda_{2} I_{2}(t) S_{2}(t)\right) \leq 0
$$

and hence, $G(t)$ is a decreasing function. By this monotonicity and Theorem 1, we conclude that $G(\infty)=\lim _{t \rightarrow \infty} G(t)$ exists and is nonnegative, and $\lim _{t \rightarrow \infty} G^{\prime}(t)=0$. This and (3.8) in turn imply that

$$
\lambda_{1} I_{1}(t) S_{1}(t)+\lambda_{2} I_{2}(t) S_{2}(t) \rightarrow 0, \text { as } t \rightarrow \infty .
$$

This limit, together with the nonnegativity of $S_{i}(t)$ and $I_{i}(t)(i=1,2)$ leads to

$$
I_{1}(t) S_{1}(t) \rightarrow 0, I_{2}(t) S_{2}(t) \rightarrow 0, \text { as } t \rightarrow \infty
$$

Now rewrite (3.3) as

$$
\frac{d}{d t} \mathbf{I}(t)=A \mathbf{I}(t)+P(t)
$$

where $P(t)=\left(p_{1}(t), p_{2}(t)\right)^{T}$ with

$$
\begin{aligned}
& p_{1}(t)=\epsilon\left(1-\alpha_{1}(\tau)\right) \lambda_{1} S_{1}(t-\tau) I_{1}(t-\tau)+\epsilon \alpha_{2}(\tau) \lambda_{2} S_{2}(t-\tau) I_{2}(t-\tau) \rightarrow 0, \\
& p_{2}(t)=\epsilon \alpha_{1}(\tau) \lambda_{1} S_{1}(t-\tau) I_{1}(t-\tau)+\epsilon\left(1-\alpha_{2}(\tau)\right) \lambda_{2} S_{2}(t-\tau) I_{2}(t-\tau) \rightarrow 0,
\end{aligned}
$$

as $t \rightarrow \infty$ by (3.10). Thus, (3.11) has

$$
\frac{d}{d t} \mathbf{X}(t)=A \mathbf{X}(t)
$$

as its limit equation for which the trivial equilibrium $(0,0)$ is globally asymptotically stable. By the theory of asymptotically autonomous systems (see, e.g., $[10,13]$ ), every solution $\mathbf{I}(t)$ of $(3.11)$ also converges to $(0,0)$ as $t \rightarrow \infty$. Thus, we have proved

Theorem 3. Let $\left(S_{1}(t), S_{2}(t), I_{1}(t), I_{2}(t)\right)$ be the solution of (2.15) with (3.1). Then, $I_{1}(t) \rightarrow 0$ and $I_{2}(t) \rightarrow 0$ as $t \rightarrow \infty$. 
Next, we address the long term behavior of $S_{1}(t)$ and $S_{2}(t)$. In the classic Kermack-McKendrick model (1.1) where the susceptible population is immediately seen to be decreasing (and bounded) implying that there is a final size $S(\infty)=\lim _{t \rightarrow \infty} S(t)$. However, for (2.15), the existence of $\lim _{t \rightarrow \infty} S_{1}(t)$ and $\lim _{t \rightarrow \infty} S_{2}(t)$ is not obvious and needs to be confirmed.

Let $F(t)=D_{S_{2}} S_{2}(t)-D_{S_{1}} S_{1}(t)$. From (2.15), we have

$$
\frac{d F}{d t}=-\left(D_{S_{1}}+D_{S_{2}}\right) F(t)+r(t)
$$

where $r(t)=D_{S_{1}} \lambda_{1} I_{1}(t) S_{1}(t)-D_{S_{2}} \lambda_{2} I_{2}(t) S_{2}(t)$. By (3.10), $r(t) \rightarrow 0$ as $t \rightarrow \infty$, and hence, equation (3.13) has

$$
\frac{d f(t)}{d t}=-\left(D_{S_{1}}+D_{S_{2}}\right) f(t)
$$

as its limit equation whose dynamics is global convergence to 0 . By the theory of asymptotically autonomous systems (see, e.g., [10,13]), $F(t)$ converges to 0 as $t \rightarrow \infty$. Note that $G(t)=$ $S_{1}(t)+S_{2}(t)$ also converges as $t \rightarrow \infty$ (see (3.8)). Now by

$$
S_{1}(t)=\frac{D_{S_{2}} G(t)-F(t)}{D_{S_{1}}+D_{S_{2}}} \quad \text { and } \quad S_{2}(t)=\frac{F(t)+D_{S_{1}} G(t)}{D_{S_{1}}+D_{S_{2}}}
$$

we conclude that both $S_{1}(\infty)=\lim _{t \rightarrow \infty} S_{1}(t)$ and $S_{2}(\infty)=\lim _{t \rightarrow \infty} S_{2}(t)$ exist. Moreover, the equation (3.15) and the limit $F(\infty)=0$ lead to

$$
S_{1}(\infty)=\frac{D_{S_{2}}}{D_{S_{1}}+D_{S_{2}}} G(\infty) \quad \text { and } \quad S_{2}(\infty)=\frac{D_{S_{1}}}{D_{S_{1}}+D_{S_{2}}} G(\infty)
$$

which determine the ratio of the two final sizes:

$$
\frac{S_{1}(\infty)}{S_{2}(\infty)}=\frac{D_{S_{2}}}{D_{S_{1}}}
$$

Summarizing the above, we have established the following

Theorem 4. Let $\left(S_{1}(t), S_{2}(t), I_{1}(t), I_{2}(t)\right)$ be the solution of (2.15) with (3.1). Then, $S_{1}(\infty)=$ $\lim _{t \rightarrow \infty} S_{1}(t)$ and $S_{2}(\infty)=\lim _{t \rightarrow \infty} S_{2}(t)$ exist and satisfy (3.17).

Although Theorems 3-4 have confirmed that the solution $\left(S_{1}(t), S_{2}(t), I_{1}(t), I_{2}(t)\right)$ of (2.15) with (3.1) converges to $\left(S_{1}(\infty), S_{2}(\infty), 0,0\right)$ with the ratio $S_{1}(\infty) / S_{2}(\infty)$ being determined by the dispersion coefficients of the susceptible sub-populations between the two patches, unfortunately, we are unable to derive an equation parallel to (1.2) that determines the final sizes $S_{1}(\infty)$ and $S_{2}(\infty)$, even implicitly. This is because, unlike (1.1) for which one can easily find a first integral, for the generalized Kermack-McKendrick model (2.15), integration becomes very difficult, if not impossible. 
Another regret for (2.15) is that we are unable to determine the patterns by which the disease dies out in both patches. This is because there are more terms, including both instantaneous and delayed terms, on the right-hand sides of the $I_{1}(t)$ and $I_{2}(t)$ equations. In the next section, we will perform some numeric simulations which show that the new model (2.15) may demonstrate very rich patterns for the disease to die out. In particular, it allows multiple outbreaks, contrasting to the simple outbreak pattern described by the classic Kermack-McKendrick model (1.1).

\section{Numeric study of disease patterns}

In this section, we numerically explore the patterns by which the disease dies out. The main focus is the impact of the latent period $\tau$ and/or dispersion rates on the outbreak patterns. For this purpose, we will discuss the following two cases: the case in which the disease does not have a latency, and the case in which the disease does have a latency. In the first case, we will observe how the dispersion of susceptible and infectious individuals affects the patterns of disease outbreak in both patches. In the latter case, we will investigate how the length of the latent period influences the disease dynamics when the dispersion rates are fixed, and how the dispersal rates of the latent individuals affect the dynamics of the disease with a fixed latent period. Finally, we will investigate numerically the joint effect of the disease latency and spatial dispersion on the disease outbreak patterns.

Since our focus is the impact of dispersion and latency, throughout this section, we set the parameters for the removal rates and disease transmission rates in both patches as follows:

$$
\sigma_{1}=0.55, \quad \sigma_{2}=0.75, \quad \lambda_{1}=0.0005, \quad \lambda_{2}=0.00025
$$

Without loss of generality, we only consider the simpler case with $d_{l}=0$ giving $\epsilon=1$. For convenience of simulations, we calculate the non-local factors $\alpha_{1}(\tau)$ and $\alpha_{2}(\tau)$ defined in (2.13) as the following more explicit formulas:

$$
\alpha_{1}(\tau)=\frac{D_{l_{1}}}{D_{l_{1}}+D_{l_{2}}}\left(1-e^{-\left(D_{l_{1}}+D_{l_{2}}\right) \tau}\right), \quad \alpha_{2}(\tau)=\frac{D_{l_{2}}}{D_{l_{1}}+D_{l_{2}}}\left(1-e^{-\left(D_{l_{1}}+D_{l_{2}}\right) \tau}\right) .
$$

\subsection{The case without latency: $\tau=0$}

When the disease has no latency, $\tau=0$ and hence $\alpha_{1}=\alpha_{2}=0$. In such a simple case, the model reduces to

$$
\left\{\begin{array}{l}
\frac{d}{d t} S_{1}(t)=D_{S_{2}} S_{2}(t)-D_{S_{1}} S_{1}(t)-\lambda_{1} I_{1}(t) S_{1}(t), \\
\frac{d}{d t} S_{2}(t)=D_{S_{1}} S_{1}(t)-D_{S_{2}} S_{2}(t)-\lambda_{2} I_{2}(t) S_{2}(t), \\
\frac{d}{d t} I_{1}(t)=-\sigma_{1} I_{1}(t)+D_{I_{2}} I_{2}(t)-D_{I_{1}} I_{1}(t)+\lambda_{1} I_{1}(t) S_{1}(t), \\
\frac{d}{d t} I_{2}(t)=-\sigma_{2} I_{2}(t)+D_{I_{1}} I_{1}(t)-D_{I_{2}} I_{2}(t)+\lambda_{2} I_{2}(t) S_{2}(t) .
\end{array}\right.
$$


We are interested in how the dispersion of susceptible and infectious individuals influences the patterns by which the disease dies out in both patches. For this purpose, we distinguish the following two cases: one is that susceptible individuals travel but infectious individuals in both patches do not travel, the other is that individuals of both classes in both patches travel.

\subsubsection{Susceptible individuals travel but infectious individuals of both patches do not travel}

This case corresponds to $D_{I_{i}}=0,(i=1,2)$ and $D_{S_{i}}>0,(i=1,2)$. In such a situation, from the third and fourth equations in (4.3), it is seen that, just as in the classical Kermack-McKendrick model, there is a critical value $S_{0}^{*}=\sigma / \lambda$ in each patch for the initial size of the susceptible class, beyond which the disease in that patch will initially experience an outbreak $(I(t)$ increases), and below which the disease initially decays. With (4.1), these two critical values are $S_{10}^{*}=\sigma_{1} / \lambda_{1}=$ 1100 and $S_{20}^{*}=\sigma_{2} / \lambda_{2}=3000$. Unlike in the classical Kermack-McKendrick model where $S(t)$ always decreases, now with the positive dispersion rates $D_{S_{i}}>0,(i=1,2), S_{1}(t)$ and/or $S_{2}(t)$ may experience some growth initially, although $S_{1}(t)+S_{2}(t)$ always decreases. This will cause changes in the disease dynamics in the two patches.

Figure 1 shows the phase portraits of $I_{i}$ vs $S_{i}, i=1,2$ for various sets of $D_{S_{1}}$ and $D_{S_{2}}$ values shown in its caption. The initial values are given as follows:

$$
S_{10}=3000, S_{20}=2500, I_{10}=10, I_{20}=50 .
$$

The ratio $d:=D_{S_{1}} / D_{S_{2}}$ describes the relative strengths of the dispersion of susceptible individuals between the two patches, and the four subfigures are results of numeric simulations obtained by varying the value of $d$ ascending order. Note that $S_{10}>S_{10}^{*}$ and $S_{20}<S_{20}^{*}$. Thus, $I_{1}(t)$ initially increases but $I_{2}(t)$ initially decreases. It is interesting to notice that when $d$ is reasonably large, $I_{2}(t)$ will also experience an outbreak before it dies out, as is shown in Figure 1-(iii)-(iv). This is because when $D_{S 1}$ is much larger than $D_{S 2}$, and hence, there will be more susceptible individuals traveling from Patch 1 to Patch 2 than from Patch 2 to Patch 1, making more available susceptible individuals in Patch 2 for infection. Therefore, such an outbreak in Patch 2 is purely caused by the unbalanced travel of susceptible individuals.

Now we change the initial distributions (4.4) in both patches to

$$
S_{10}=3000, S_{20}=3500, I_{10}=10, I_{20}=50,
$$

such that both $I_{1}(t)$ and $I_{2}(t)$ increase initially. This time, varying the ratio $d:=D_{S_{1}} / D_{S_{2}}$ does not change the patterns of $I_{1}$ and $I_{2}$ (one outbreak), but only causes some changes to the patterns of $S_{1}$ and $S_{2}$. However, simulations show that the change of $d$ does affect the magnitudes of the outbreaks in the two patches, as shown in Figure 2.

\subsubsection{Susceptible and infectious individuals in both patches travel}

In this case, common sense seems to suggest the assumption $D_{S_{i}}>D_{I_{i}},(i=1,2)$, since infectious individuals have a lower level of travel intention. We choose $D_{I_{2}}=0.10>D_{I_{1}}=0.09$. From 
the third and fourth equations in (4.3), larger $D_{I_{2}}$ should boost $I_{1}$ initially but should not favor $I_{2}$. Numeric simulations show that, again as in Section 4.1.1, larger values of $D_{S_{1}}$ (relative to $D_{S_{2}}$ ) can cause an outbreak of the disease in Patch 2. See Figure 3 for simulations of (4.3) with initial values (4.4).

Figure 4 are some numeric simulation results for (4.3) but with initial distribution (4.5). Comparing the Figure 4-(ii) with Figure 2-(ii), we see that in the former, $I_{2}(t)$ first experiences a quiescent period before its outbreak. This is due to the interplay of $D_{I_{i}}$ and $D_{S_{i}}, i=1.2$.

\subsection{The cases with latency $\tau>0$}

In this case, the model is a system of delay differential equations. We mainly examine the impact of the latent delay $\tau$ and the dispersion rates $D_{l_{i}}, i=1,2$, during the latent period. To this end, we first fix $\tau>0, D_{S_{i}}$, and $D_{I_{i}}, i=1,2$, at some levels, and observe how the disease patterns will change as $D_{l_{i}}, i=1,2$, vary. Then, we will fix the dispersion rates at certain levels and numerically investigate the impact of the latent delay $\tau$ on the disease dynamics. When we set values of the dispersion rates for simulations, for realistic reasons, we abide by the restriction $D_{I_{i}} \leq D_{l_{i}} \leq D_{S_{i}}, \quad i=1,2$.

For simulation convenience, we introduce the ratio $l:=D_{l_{1}} / D_{l_{2}}$, which measures the relative dispersion strength of latent individuals in Patch 1 to Patch 2.

\subsubsection{For fixed $\tau>0$ consider the impact of various dispersion rates}

In this subsection, we fixed the disease latency at $\tau=10$. Figure 5 corresponds to the case in which the infectious individuals do not travel and Figure 6 are simulation results of the case when the infectious individuals travel but at a lower rate than the latent individuals do. In both figures, the initial functions are taken constant functions with values given by $(4.5)$ on $[-\tau, 0]$.

Figure 5-(i) shows the disease dynamics in the absence of dispersion (two patches are disconnected) where the disease experiences one outbreak in both patches before dying out, although the outbreak speed in Patch 1 fluctuates at several points. The incorporation of dispersion for latent and susceptible classes has an immediate impact on the pattern in Patch 2: $I_{2}(t)$ changes from being initially increasing to being initial decreasing before going to an outbreak, as are shown in Figure 5-(ii)-(iii)-(iv). Such a change in the initial disease dynamics may easily give one a wrong impression that the disease starts dying out, and hence is worth particular attention and warning. Another important finding is that the dispersion strengths of latent individuals may cause multiple outbreaks, as is shown in Figure 5-(iv) where the disease evolves into two outbreaks in both patches from the previous one outbreak.

In Figure 6-(ii)-(iii)-(iv), different from Figure 5, $D_{I_{1}}$ and $D_{I_{2}}$ are fixed at positive values $D_{I_{1}}=$ 0.1 and $D_{I_{2}}=0.09$. Similar phenomena to that in Figure 5 are observed, but the two-outbreak pattern develops earlier than that in Figure 5 as $l$ is increased (it appears for $l=D_{l_{1}} / D_{l_{2}}=1$ in Figure 6 but not in Figure 5). This implies that $D_{I_{i}}, i=1,2$, also play a role in causing multiple outbreaks. 


\subsubsection{Impact of the length of the latent period}

In this subsection, we numerically investigate the influence of the magnitude of the disease latency on the pattern of the disease outbreak. For this purpose, we fix the dispersion rates at several levels as in the previous subsections, indicated as subfigures (i)-(iv) as before, and observe the changes of the disease patterns for three values of $\tau: \tau=5,10,15$. The initial functions are taken constant functions with values given by (4.5) on $[-\tau, 0]$.

Figure 7-(i) and Figure 8-(i) reveals that even if the two patches are isolated, larger latency tends to cause multiple outbreaks and in the mean time, decrease the outbreak sizes. Figure 7-(ii)(iii)-(iv) show that the two patches may have different number of outbreaks, due to the interaction of $D_{S_{1}}$ and $D_{S_{2}}$. For example, in Figure 7-(ii) and for $\tau=15$, Patch 2 only experiences one outbreak while Patch 1 suffers two outbreaks.

Figure 8 aims at comparing the results of various values of the ratio $l=D_{l_{1}} / D_{l_{2}}$. Interestingly, when $D_{l_{i}}$ and $D_{I_{i}}$ are turned on, the inconsistency in the numbers of outbreaks in the two patches disappears, as is shown in Figure 8-(ii)-(iii)-(iv) (comparing with 7-(ii)-(iii)-(iv)). Also in Figure 8-(ii)-(iii)-(iv), for the given values of the parameters and initial values, a relatively longer period of quiescence for the disease in Patch 2 is observed, after an initial decrease and before its first outbreak. This quiescent period seems to increase as $\tau$ and $l=D_{l_{1}} / D_{l_{2}}$ increase.

Another observation from Figure 7 and 8 is that although a larger latent period can cause multiple outbreaks, it also decreases the sizes of outbreaks. The dispersion rates also affect the outbreak sizes. Analytically determining these sizes seem to be very difficult, if not impossible.

At last, we can also numerically confirm the conclusion on the final sizes in the two patches given in Theorem 4. For example, consider the model with parameters and initial values leading to Figure 7. At $t=200, I_{i}(200) \approx 0$. For $d=0.1$, we have $S_{1}(200)=102.6957, S_{2}(200)=10.2696$, and hence $S_{2}(200) / S_{1}(200)=0.1000=d$; for $d=1$, we have $S_{1}(200)=385.8986, S_{2}(200)=$ 385.8986 , and $S_{2}(200) / S_{1}(200)=1$; for $d=2$, we have $S_{1}(200)=576.4, S_{2}(200)=1152.7$, and $S_{2}(200) / S_{1}(200)=2.0000$, all confirming the relation $S_{2}(\infty) / S_{1}(\infty)=D_{S_{1}} / D_{S_{2}}=d$. For the parameters and initial values leading to Figure $8, d=D_{S_{1}} / D_{S_{2}}=0.45 / 0.5=0.9000$ is fixed in Figure 8-(ii)-(iii)-(iv). Now for $l=0.1, S_{1}(200)=295.3050$ and $S_{2}(200)=265.7736$ giving $S_{2}(200) / S_{1}(200)=0.9000=d$; for $l=0.5, S_{1}(200)=382.4305$ and $S_{2}(200)=344.1852$ also giving $S_{2}(200) / S_{1}(200)=0.9000=d$; for $l=1.5, S_{1}(200)=662.5170$ and $S_{2}(200)=596.2653$ again giving $S_{2}(200) / S_{1}(200)=0.9000=d$. 

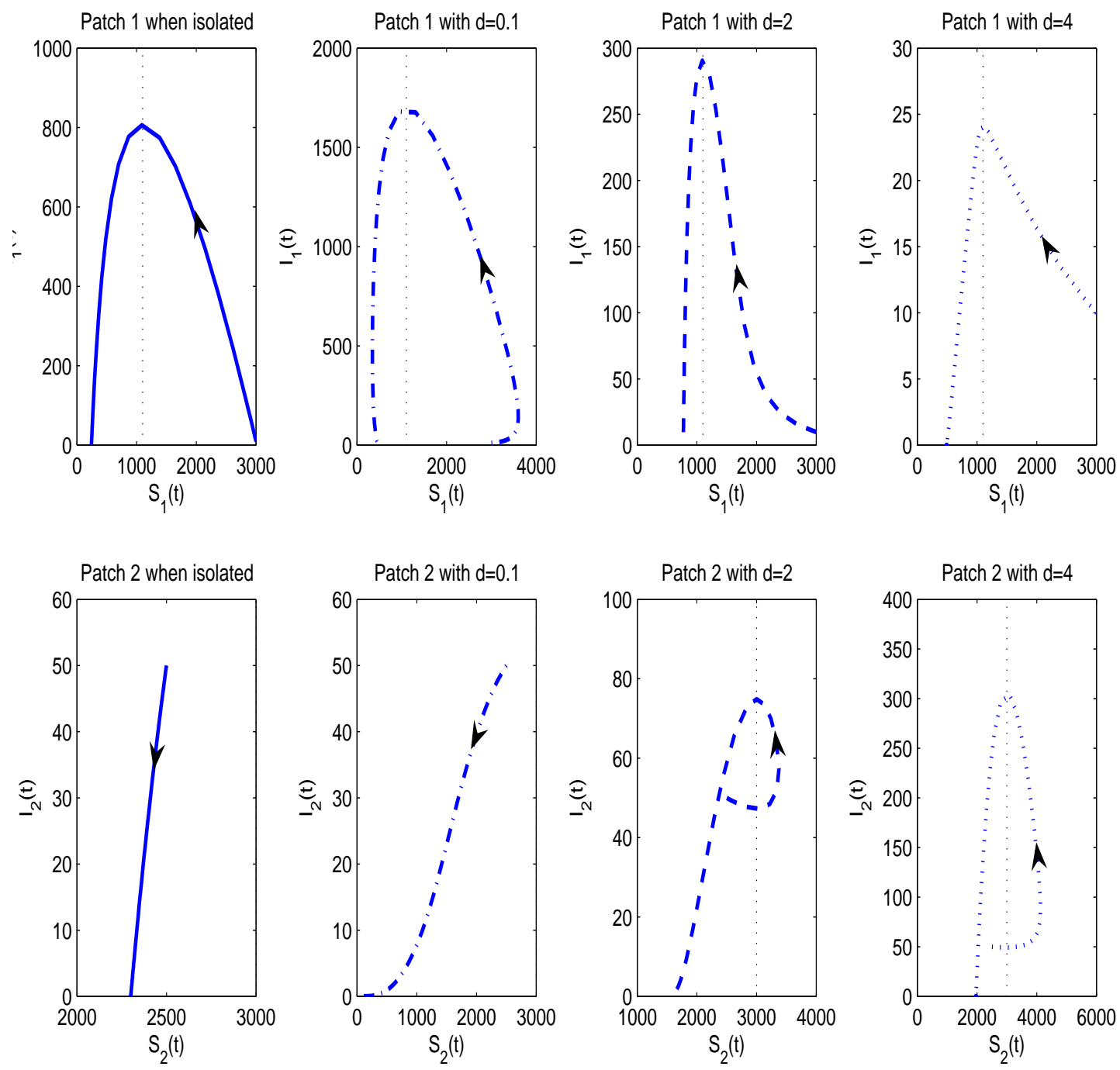

(i)

(ii)

(iii)

(iv)

Figure 1: The phase portraits of $I_{i}(t)$ vs $S_{i}(t)(i=1,2)$ for (4.3) with (4.1), $D_{I_{i}}=0, i=1,2$ and (i) $D_{S_{1}}=0, D_{S_{2}}=0$; (ii) $D_{S_{1}}=0.02, D_{S_{2}}=0.2$; (iii) $D_{S_{1}}=0.4, D_{S_{2}}=0.2$; (iv) $D_{S_{1}}=0.8, D_{S_{2}}=$ 0.2 . Initial values are given by (4.4). 

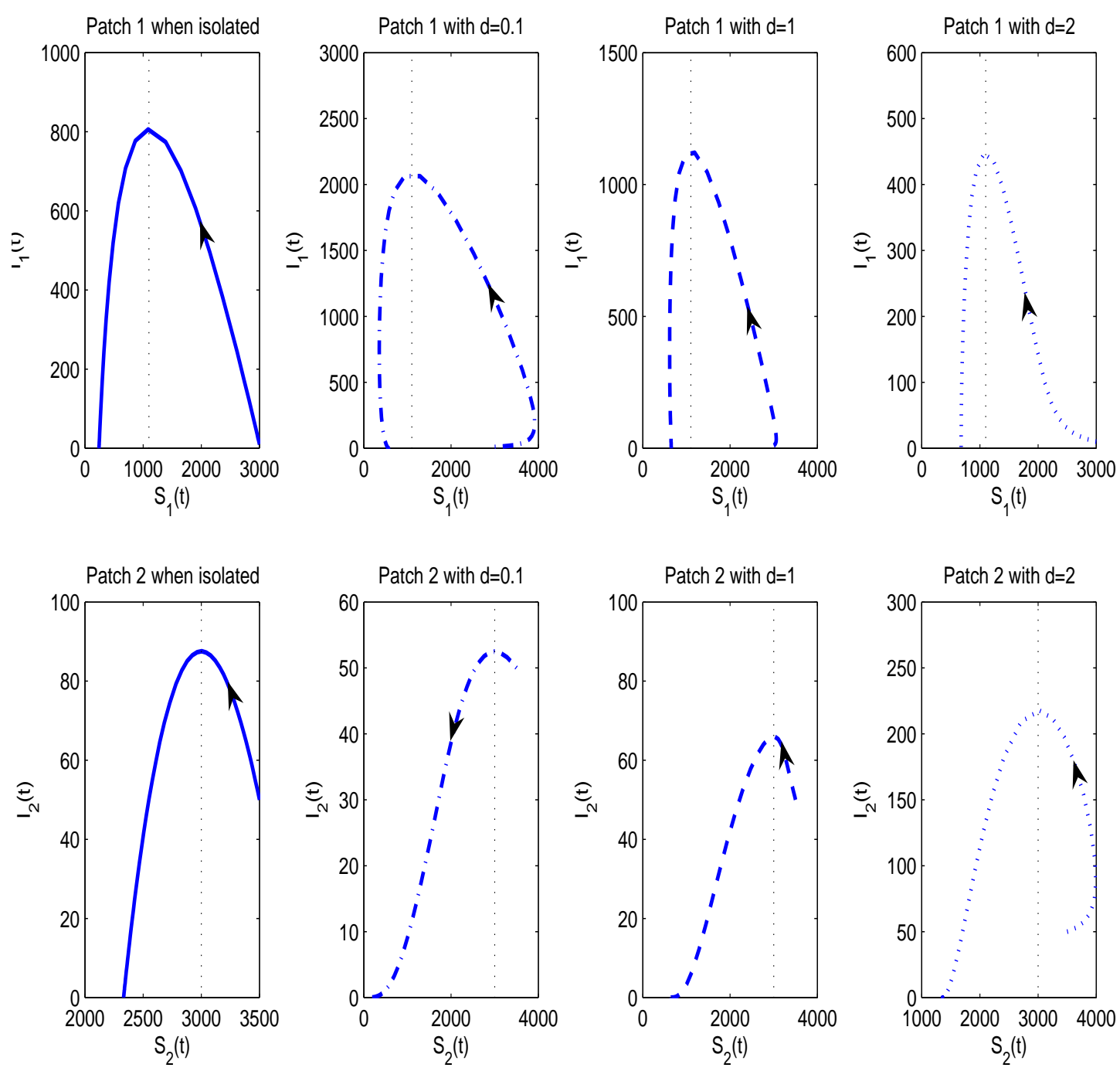

(i)

(ii)

(iii)

(iv)

Figure 2: The phase portraits of $I_{i}(t)$ vs $S_{i}(t)(i=1,2)$ for (4.3) with (4.1), $D_{I_{i}}=0, i=1,2$ and (i) $D_{S_{1}}=0, D_{S_{2}}=0$; (ii) $D_{S_{1}}=0.02, D_{S_{2}}=0.2$; (iii) $D_{S_{1}}=0.2, D_{S_{2}}=0.2$; (iv) $D_{S_{1}}=0.4, D_{S_{2}}=$ 0.2 . Initial values are given by (4.5). 

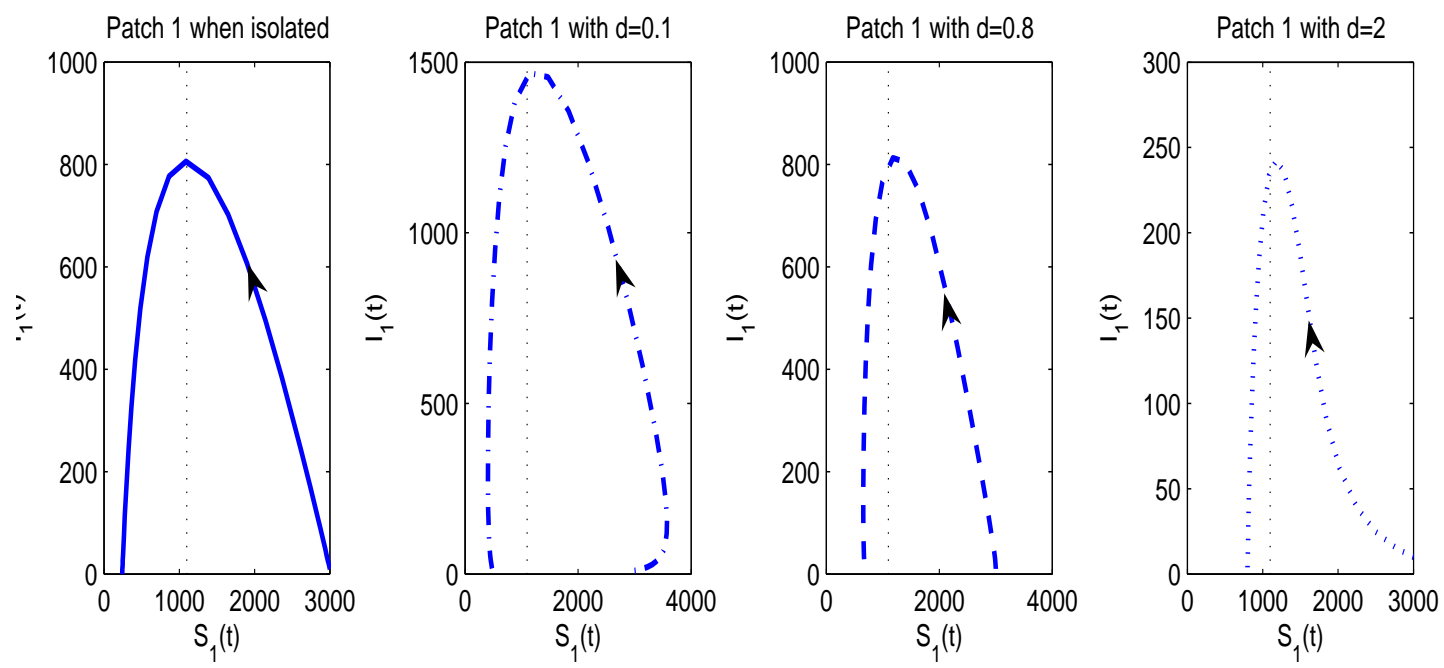

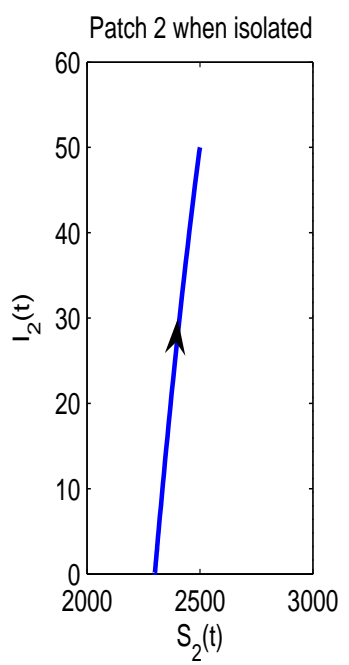

(i)

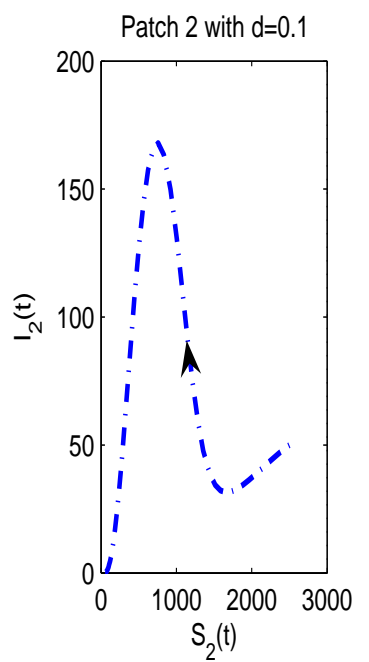

(ii)

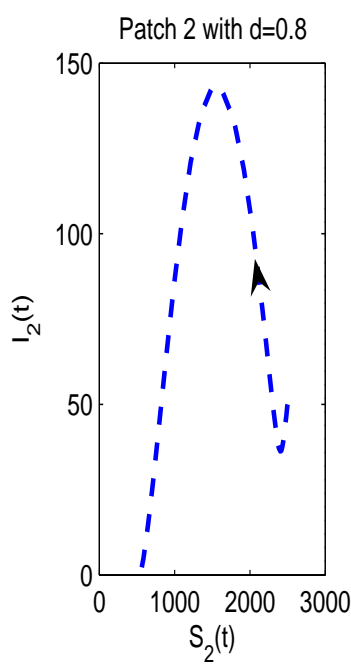

(iii)

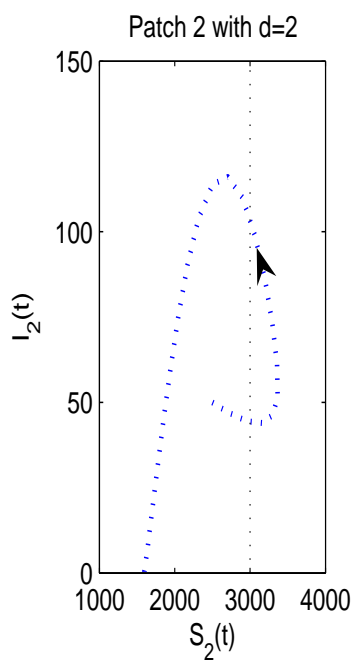

(iv)

Figure 3: The phase portraits of $I_{i}(t)$ vs $S_{i}(t)(i=1,2)$ for (4.3) with (4.1) and (i) $D_{S_{1}}=0, D_{S_{2}}=$ $0, D_{I_{1}}=0, D_{I_{2}}=0$; (ii) $D_{S_{1}}=0.02, D_{S_{2}}=0.2, D_{I_{1}}=0.09, D_{I_{2}}=0.1$; (iii) $D_{S_{1}}=0.16, D_{S_{2}}=$ $0.2, D_{I_{1}}=0.09, D_{I_{2}}=0.1$; (iv) $D_{S_{1}}=0.4, D_{S_{2}}=0.2, D_{I_{1}}=0.09, D_{I_{2}}=0.1$. Initial values are given by (4.4). 

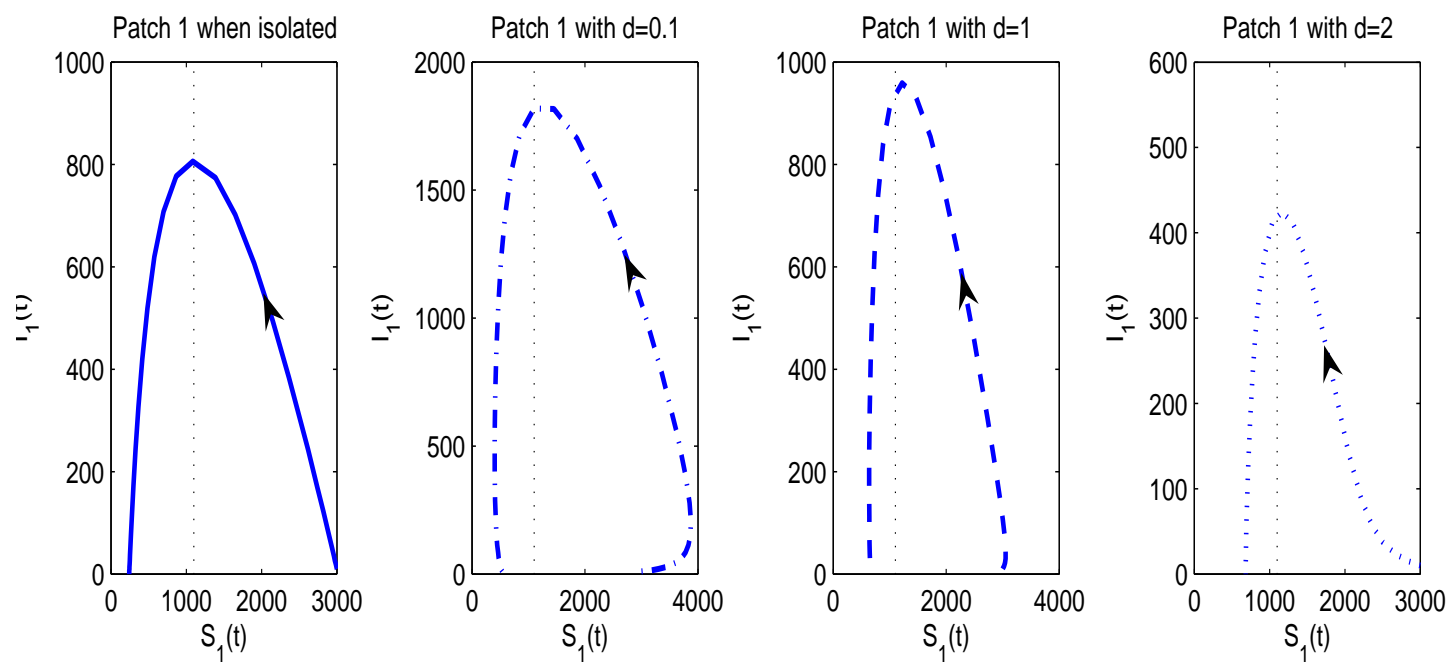

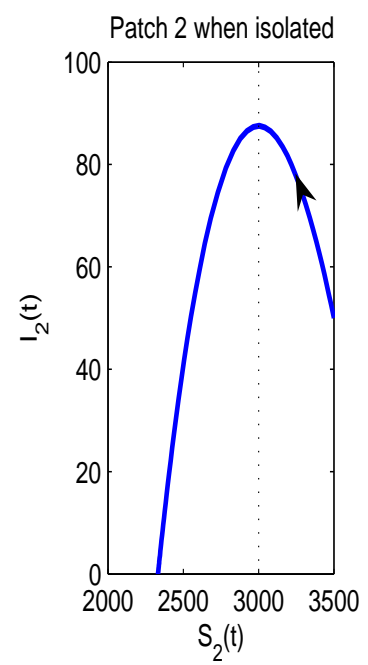

(i)

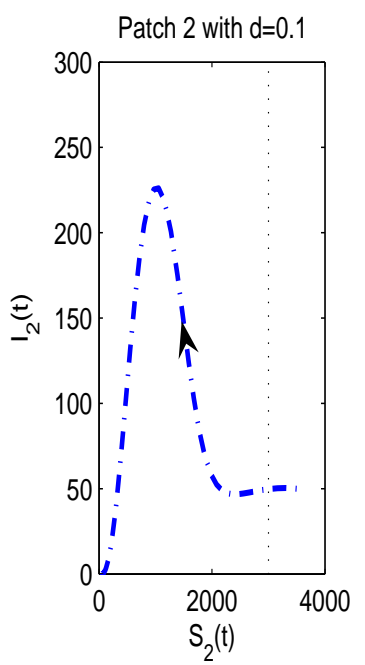

(ii)

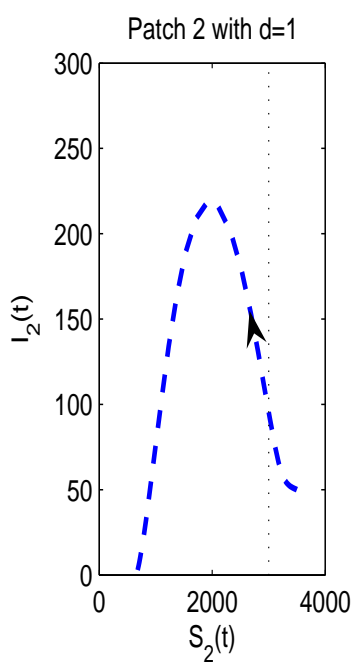

(iii)

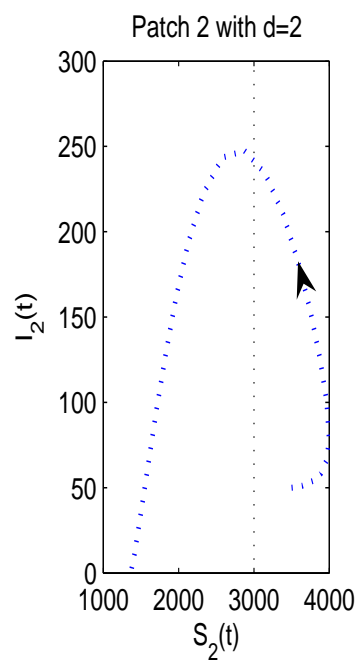

(iv)

Figure 4: The phase portraits of $I_{i}(t)$ vs $S_{i}(t)(i=1,2)$ for (4.3) with (4.1) and (i) $D_{S_{1}}=0, D_{S_{2}}=$ $0, D_{I_{1}}=0, D_{I_{2}}=0$; (ii) $D_{S_{1}}=0.02, D_{S_{2}}=0.2, D_{I_{1}}=0.09, D_{I_{2}}=0.1$; (iii) $D_{S_{1}}=0.2, D_{S_{2}}=$ $0.2, D_{I_{1}}=0.09, D_{I_{2}}=0.1$; (iv) $D_{S_{1}}=0.4, D_{S_{2}}=0.2, D_{I_{1}}=0.09, D_{I_{2}}=0.1$. Initial values are given by (4.5). 

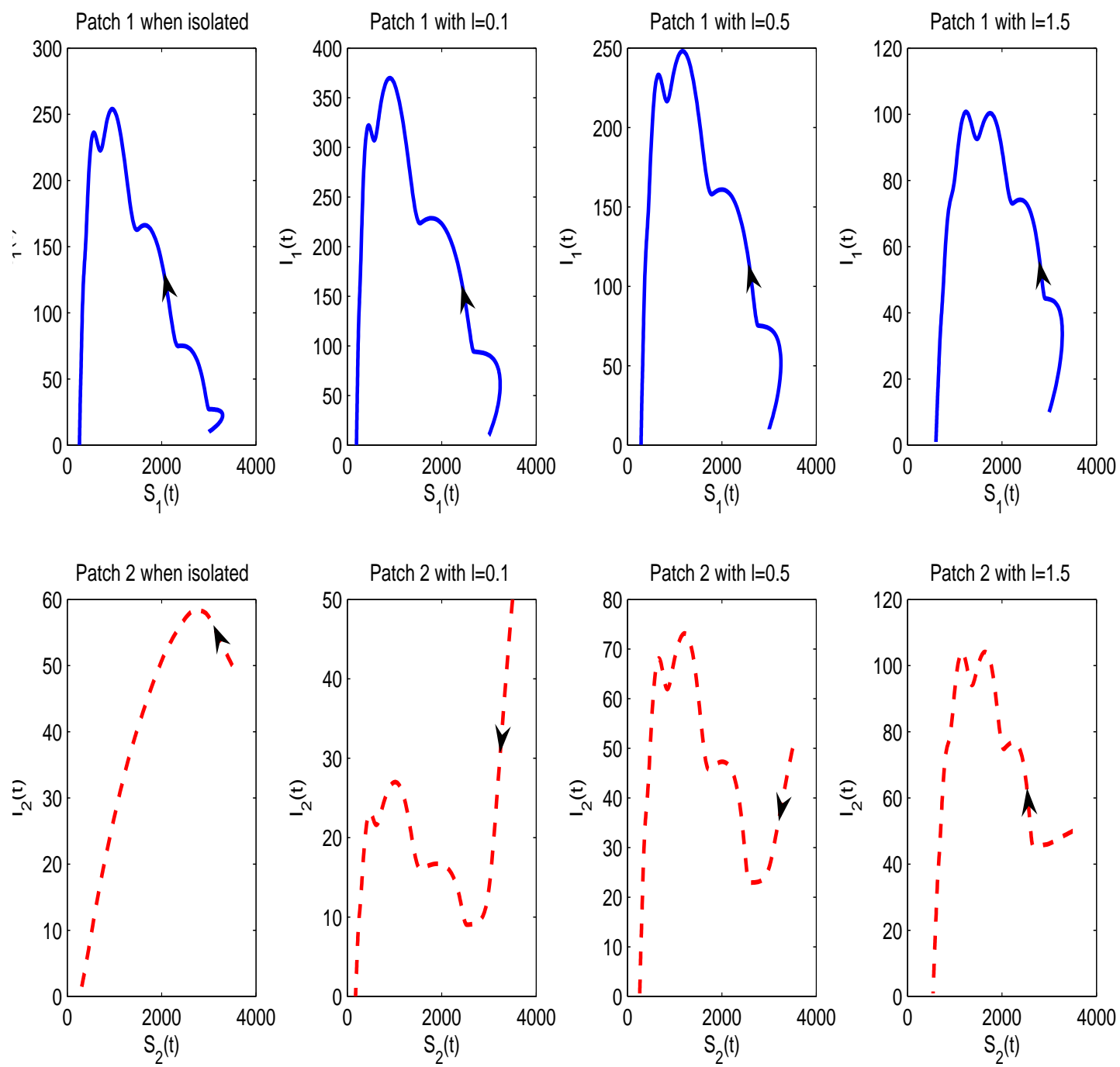

(i)

(ii)

(iii)

(iv)

Figure 5: The phase portraits of $I_{i}(t)$ vs $S_{i}(t)(i=1,2)$ for (2.15) with (4.1), $\epsilon=1, D_{I_{i}}=0, i=1,2$ and (i) $D_{S_{1}}=0, D_{S_{2}}=0, D_{l_{1}}=0, D_{l_{2}}=0$; (ii) $D_{S_{1}}=0.45, D_{S_{2}}=0.5, D_{l_{1}}=0.03, D_{l_{2}}=0.3$; (iii) $D_{S_{1}}=0.45, D_{S_{2}}=0.5, D_{l_{1}}=0.15, D_{l_{2}}=0.3$; (iv) $D_{S_{1}}=0.45, D_{S_{2}}=0.5, D_{l_{1}}=0.45, D_{l_{2}}=0.3$. Initial values are given by (4.5). 

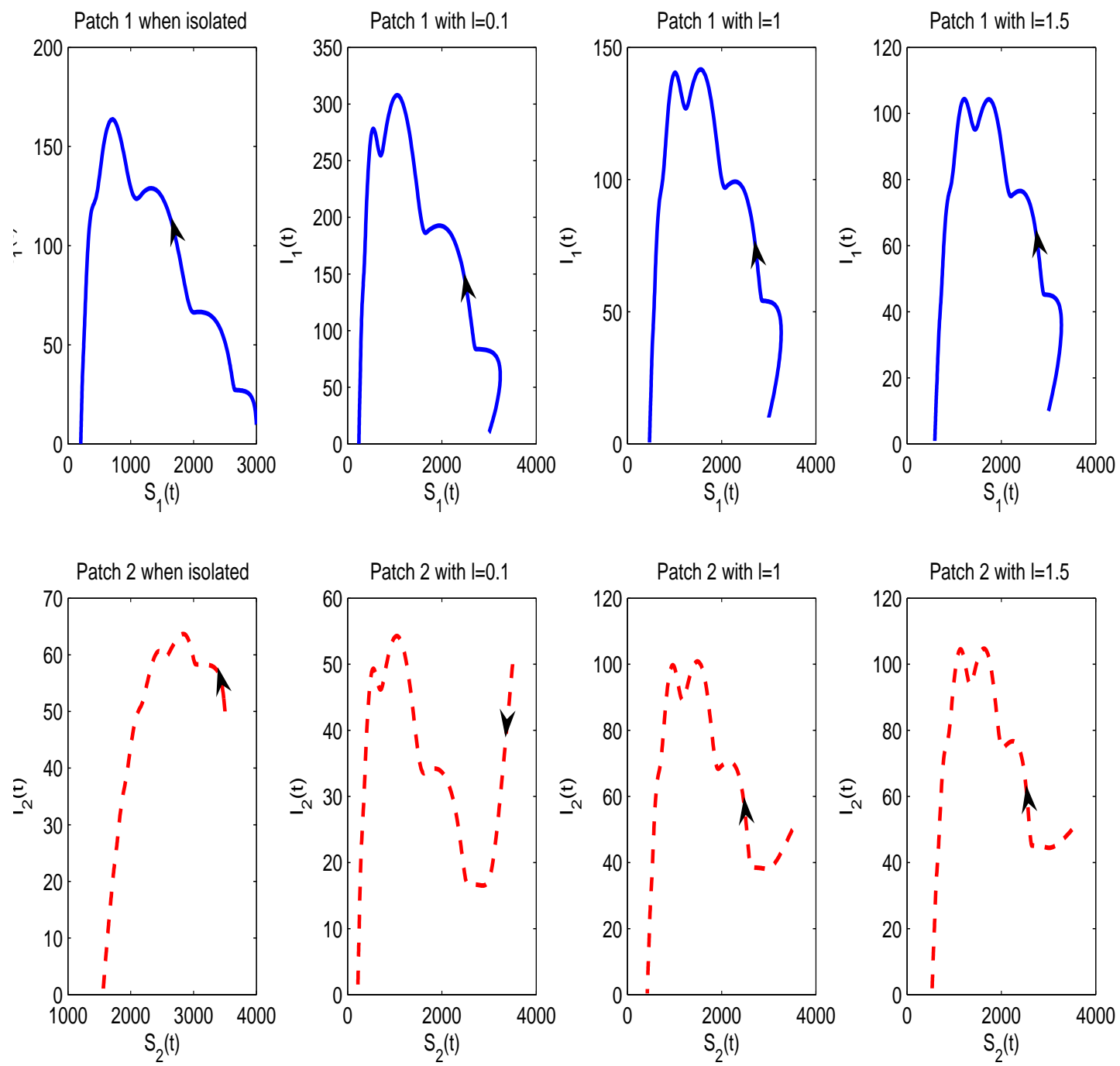

(i)

(ii)

(iii)

(iv)

Figure 6: The phase portraits of $I_{i}(t)$ vs $S_{i}(t)(i=1,2)$ for (2.15) with (4.1), $\epsilon=1$, and (i) $D_{S_{1}}=$ $0, D_{S_{2}}=0, D_{l_{1}}=0, D_{l_{2}}=0, D_{I_{1}}=0, D_{I_{2}}=0$; (ii) $D_{S_{1}}=0.45, D_{S_{2}}=0.5, D_{l_{1}}=0.03, D_{l_{2}}=$ $0.3, D_{I_{1}}=0.09, D_{I_{2}}=0.1$; (iii) $D_{S_{1}}=0.45, D_{S_{2}}=0.5, D_{l_{1}}=0.3, D_{l_{2}}=0.3, D_{I_{1}}=0.09, D_{I_{2}}=$ 0.1 ; (iv) $D_{S_{1}}=0.45, D_{S_{2}}=0.5, D_{l_{1}}=0.45, D_{l_{2}}=0.3, D_{I_{1}}=0.09, D_{I_{2}}=0.1$. Initial values are given by (4.5). 

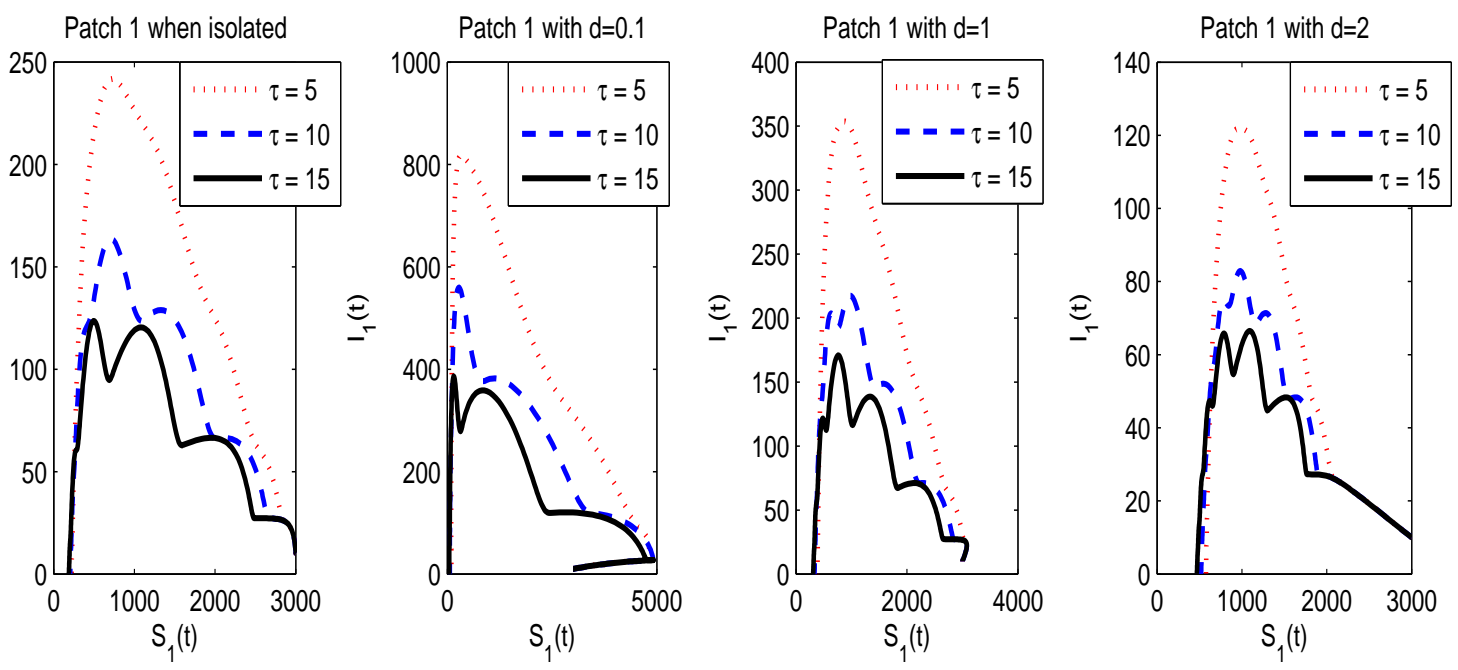

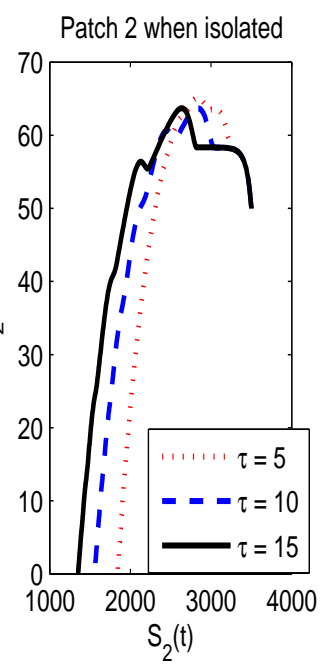

(i)

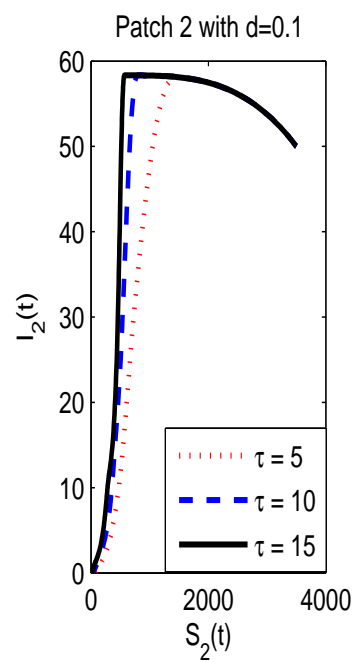

(ii)

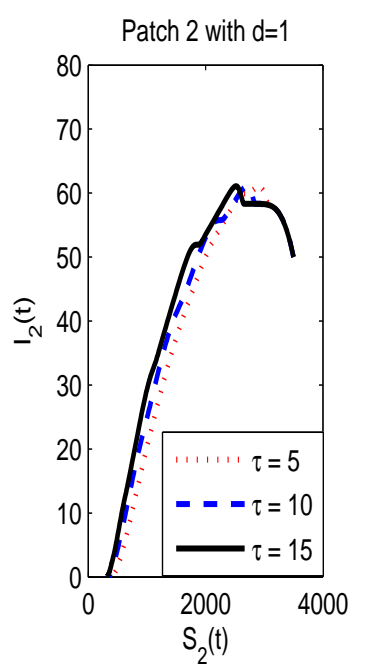

(iii)

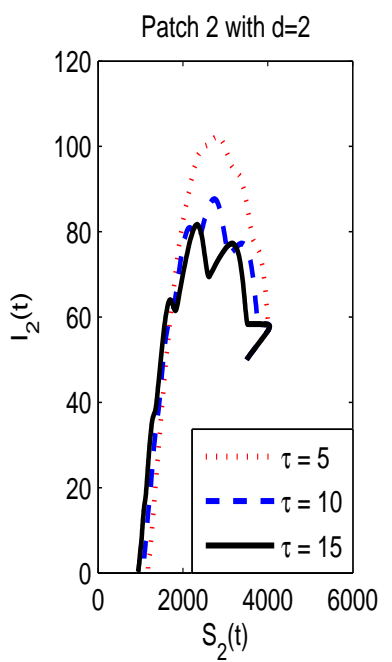

(iv)

Figure 7: The phase portrait of $I_{i}(t)$ vs $S_{i}(t)(i=1,2)$ for (2.15) with (4.5), $\epsilon=1, D_{l_{1}}=D_{l_{2}}=D_{I_{1}}=$ $D_{I_{2}}=0$ and (i) $D_{S_{1}}=0, D_{S_{2}}=0$; (ii) $D_{S_{1}}=0.02, D_{S_{2}}=0.2$; (iii) $D_{S_{1}}=0.2, D_{S_{2}}=0.2$; (iv) $D_{S_{1}}=0.4, D_{S_{2}}=0.2$. 

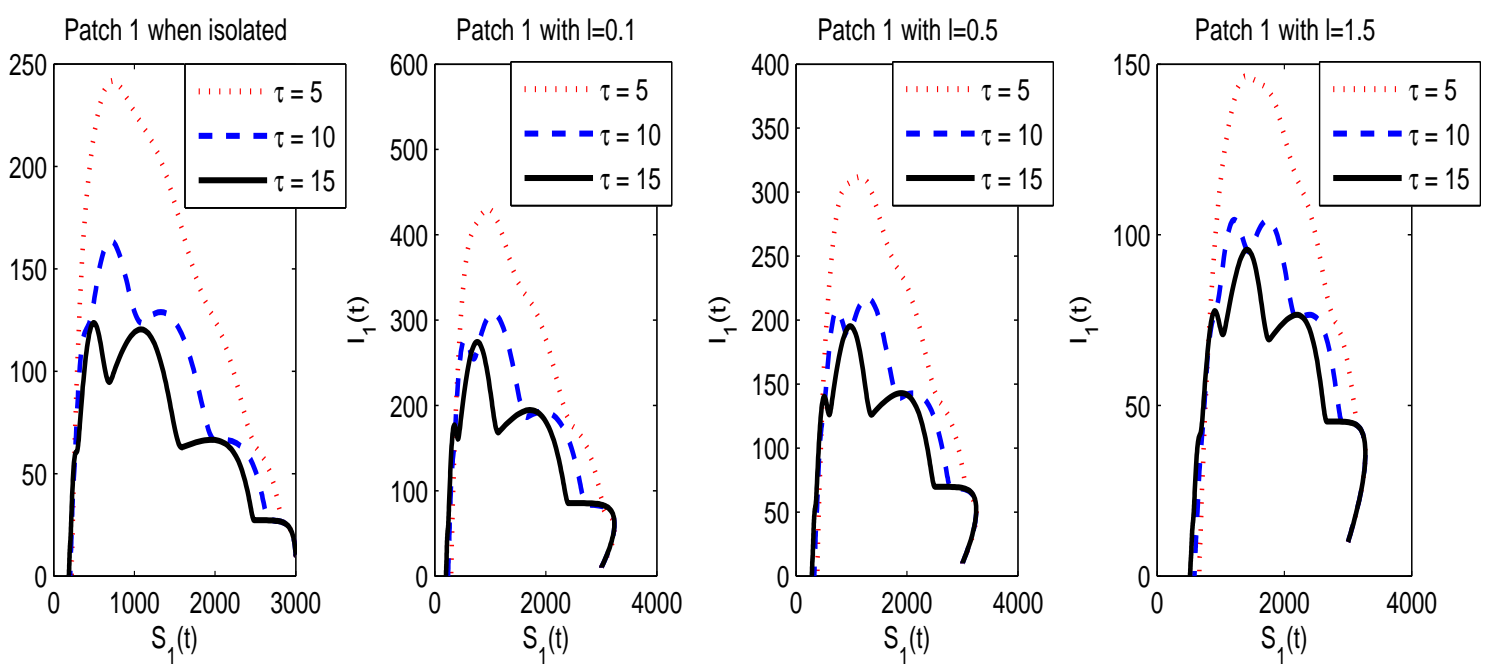

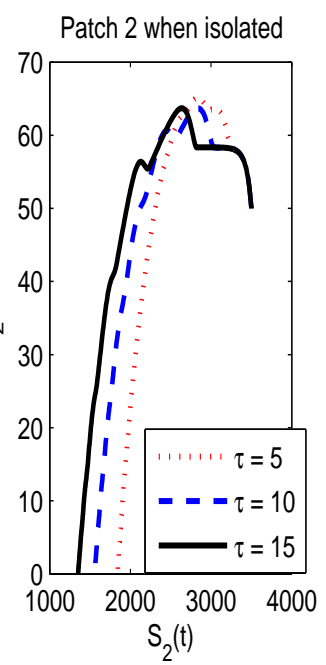

(i)

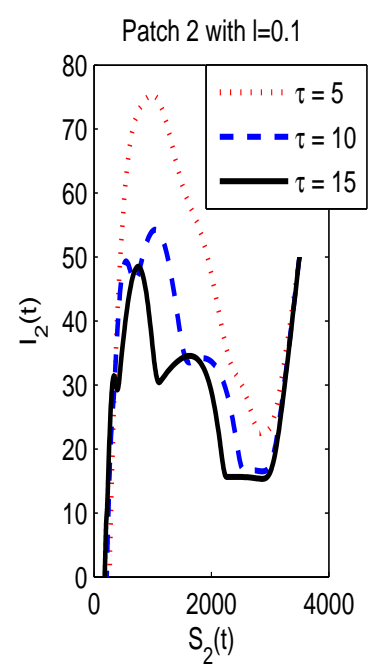

(ii)

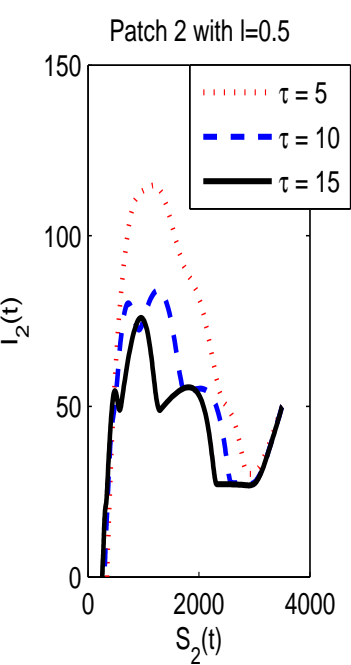

(iii)

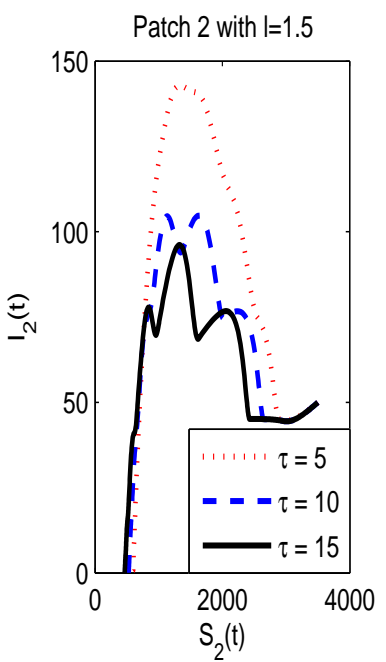

(iv)

Figure 8: The phase portrait of $I_{i}(t)$ vs $S_{i}(t)(i=1,2)$ with (4.5), $\epsilon=1$, and (i) $D_{S_{1}}=0, D_{S_{2}}=$ $0, D_{l_{1}}=0, D_{l_{2}}=0, D_{I_{1}}=0, D_{I_{2}}=0$; (ii) $D_{S_{1}}=0.45, D_{S_{2}}=0.5, D_{l_{1}}=0.03, D_{l_{2}}=0.3, D_{I_{1}}=$ $0.09, D_{I_{2}}=0.1$; (iii) $D_{S_{1}}=0.45, D_{S_{2}}=0.5, D_{l_{1}}=0.15, D_{l_{2}}=0.3, D_{I_{1}}=0.09, D_{I_{2}}=0.1$; (iv) $D_{S_{1}}=0.45, D_{S_{2}}=0.5, D_{l_{1}}=0.45, D_{l_{2}}=0.3, D_{I_{1}}=0.09, D_{I_{2}}=0.1$. 


\section{Conclusion and discussion}

In this paper, we have derived a new epidemic model to describe the dynamics of diseases with a fixed latency in a two-patch environment. Starting from the classic Kermack-McKendrick model, making use of a fundamental partial differential equation for the evolution of diseases with infection age and time, and tracking the dispersal of latent individuals, we have obtained a model in the form of a system of delay differential equations which, in addition to the linear dispersion terms, contains non-local infection terms. The patches can be communities, cities, regions and even countries; and the population dispersal among patches can be interpreted as the movements by which people travel or migrate between patches.

For this new model, we have verified the positivity and boundedness of the solutions to the system. Since the model does not consider a demographic structure for the population, as in the classical Kermack-McKendrick SIR model, we have shown that this new model also does not allow the disease to persist, meaning that the disease eventually dies out in both patches. In such a situation, final sizes $S_{1}(\infty)$ and $S_{2}(\infty)$, as well as the patterns by which the disease dies out in the two patches stand out as important issues for the model. For the former, unfortunately we can not derive equations similar to (1.3) for the classical Kermack-McKendrick SIR model that can completely determine the final sizes in the two patches. However, we have proved that the ratio of the two final sizes for the new model (2.15) is totally determined by the ratio of the dispersion rates of the susceptible individuals between the two patches. An immediate implication of this ratio result is that restricting travel from Patch 1 to Patch 2 (decreasing $D_{S_{1}}$ ) will increase $S_{1}(\infty)$ and hence will eventually benefit Patch 1. A similar conclusion applies to the consequence of restricting travel from Patch 2 to Patch 1 . This very well justifies the necessity of banning of travel to those disease areas by a city or a country.

Theoretically determining the patterns by which the disease dies out for this new model does not seem to be possible, due to the inclusion of both the latent delay and the non-local infection terms in the model. We have numerically studied this problem by examining the effect of the length of disease latency and the strengths of spatial dispersion of individuals in different classes, particularly the impact of the latency and non-locality caused by the mobility of the latent individuals on the disease dynamics. In general, the impact is complicated. We have numerically observed that (i) either the dispersion or the disease latency can cause multiple outbreaks; (ii) the number of outbreaks in the two patches can be different due to unbalanced travels; (iii) a larger latency tends to cause more outbreaks, but in the mean time, decrease the maximum outbreak sizes; (iv) relatively larger values of $D_{S_{1}}\left(D_{S_{2}}\right)$ tend to raise the maximum outbreak sizes in Patch 2 (Patch 1). These findings are in strong contrast to the disease dynamics of the classical Kermack-McKendrick SIR model (1.1). While the length of latent period is disease specific and nothing can be done to it, the dispersion rates are controllable via issuing restrictions or bans on the travels between patches. Our model and the results about the model provide some insights into how to avoid multiple outbreaks and how to predict and control the maximum outbreak sizes in the two patches.

We point out that in our new model (2.15), we have omitted the demographic structure by ignoring the recruitment (including births) and the natural deaths. This is reasonable for a fast dis- 
ease, since in such a case the disease mean life time is much shorter than the mean life expectation of the population. For a disease with longer life time, this model becomes unrealistic and modifications are needed to include the demographic structure. In a forthcoming paper, we extend the model (2.15) to one whose underlying population model (an equation that governs the population growth in the absence of disease) supports a globally asymptotically stable positive equilibrium. In such a situation, the disease free equilibrium may or may not be stable, and the basic reproduction number is closely related to the stability of this disease free equilibrium as well as to the existence of an endemic equilibrium. Along this line, there have been extensive works on epidemic models on patch environments; see e.g., [2, 3, 8, 12, 14, 15, 16] and the references therein. However, to the authors' best knowledge, none of them considers the non-local infections caused by a fixed latent time and the mobility of individuals during this latent period.

\section{Acknowledgements}

This research was supported by NSERC, by NCE-MITACS of Canada and by PREA of Ontario.

\section{References}

[1] R. M. Anderson, R. M. May. Infectious diseases of humans: dynamics and control, Oxford University Press, Oxford, UK, 1991.

[2] J. Arino, P. van den Driessche. A multi-city epidemic model. Math. Popul. Stud., 10 (2003), 175-193.

[3] J. Arino, P. van den Driessche. The basic reproduction number in a multi-city compartmental epidemic model. LNCIS, 294 (2003), 135-142.

[4] F. Brauer. Some simple epidemic models. Math. Biosci. Engin., 3 (2006), 1-15.

[5] O. Diekmann, J. A. P. Heesterbeek. Mathematical epidemiology of infectious diseases: model building, analysis and interpretation. Wiley, 2000.

[6] J. K. Hale, S. M. Verduyn Lunel. Introduction to functional differential equations. SpringVerlag, New York, 1993.

[7] W. O. Kermack, A. G. McKendrick. A contribution to the mathematical theory of epidemics. Proc. Royal Soc. London, 115 (1927), 700-721.

[8] Y.-H. Hsieh, P. van den Driessche, L. Wang. Impact of travel between patches for spatial spread of disease. Bull. Math. Biol., 69 (2007), 1355-1375.

[9] J. A. J. Metz, O. Diekmann. The dynamics of physiologically structured populations. Springer-Verlag, New York, 1986. 
[10] K. Mischaikow, H. Smith, H. R. Thieme. Asymptotically autonomous semiflows: chain recurrence and Lyapunov functions. Trans. Amer. Math. Soc., 347 (1995), 1669-1685.

[11] J. D. Murray. Mathematical biology. 3rd ed., Springer-Verlag, New York, 2002.

[12] M. Salmani, P. van den Driessche. A model for disease transmission in a patchy environment. Disc. Cont. Dynam. Syst. Ser. B, 6 (2006), 185-202.

[13] H. R. Thieme, C. Castillo-Chavez. Asymptotically autonomous epidemic models, in Mathematical Population Dynamics: Analysis of Heterogeneity, Vol. 1: Theory of Epidemics (O. Arino, D. Axelrod, M. Kimmel, M. Langlais eds.), pp. 33-50, Wuerz, 1995.

[14] W. Wang, X.-Q. Zhao. An epidemic model in a patchy environment. Math. Biosci., 190 (2004), 97-112.

[15] W. Wang, X.-Q. Zhao. An age-structured epidemic model in a patchy environment. SIAM J. Appl. Math., 65 (2005), 1597-1614.

[16] W. Wang, X.-Q. Zhao. An epidemic model with population dispersal and infection period. SIAM J. Appl. Math., 66 (2006), 1454-1472. 